University of Nebraska - Lincoln

DigitalCommons@University of Nebraska - Lincoln

\title{
Kinetics of formation of twinned structures under L10-type orderings in alloys
}

Kirill D. Belashchenko

'Kurchatov Institute' Russian Research Centre, belashchenko@unl.edu

I R Pankratov

'Kurchatov Institute' Russian Research Centre

G D Samolyuk

'Kurchatov Institute' Russian Research Centre

V G Vaks

'Kurchatov Institute' Russian Research Centre, vaks@mbslab.kiae.ru

Follow this and additional works at: https://digitalcommons.unl.edu/physicsbelashchenko

Belashchenko, Kirill D.; Pankratov, I R; Samolyuk, G D; and Vaks, V G, "Kinetics of formation of twinned structures under L10-type orderings in alloys" (2002). Kirill Belashchenko Publications. 6.

https://digitalcommons.unl.edu/physicsbelashchenko/6

This Article is brought to you for free and open access by the Research Papers in Physics and Astronomy at DigitalCommons@University of Nebraska - Lincoln. It has been accepted for inclusion in Kirill Belashchenko Publications by an authorized administrator of DigitalCommons@University of Nebraska - Lincoln. 


\title{
Kinetics of formation of twinned structures under L1 ${ }_{0}$-type orderings in alloys
}

\author{
K D Belashchenko ${ }^{1}$, I R Pankratov, G D Samolyuk ${ }^{1}$ and V G Vaks
}

'Kurchatov Institute’ Russian Research Centre, Moscow 123182, Russia

Received 23 August 2001, in final form 14 November 2001

Published 21 December 2001

Online at stacks.iop.org/JPhysCM/14/565

\begin{abstract}
The earlier-developed master equation approach and kinetic cluster methods are applied to study the kinetics of $\mathrm{L}_{0}$-type orderings in alloys, including the formation of twinned structures characteristic of cubic-tetragonal-type phase transitions. A microscopical model of interatomic deformational interactions is suggested which generalizes a similar model of Khachaturyan for dilute alloys to the physically interesting case of concentrated alloys. The model is used to simulate $\mathrm{A} 1 \rightarrow \mathrm{L}_{0}$ transformations after a quench of an alloy from the disordered $\mathrm{A} 1$ phase to the single-phase $\mathrm{L} 1_{0}$ state for a number of alloy models with different chemical interactions, temperatures, concentrations, and tetragonal distortions. We find a number of peculiar features in both transient microstructures and transformation kinetics, many of them agreeing well with experimental data. The simulations also demonstrate the phenomenon of an interaction-dependent alignment of antiphase boundaries in nearly equilibrium twinned bands which seems to be observed in some experiments.
\end{abstract}

\section{Introduction}

Studies of microstructural evolution under alloy phase transformations from the disordered FCC phase (A1 phase) to the CuAu I-type ordered tetragonal phase ( $\mathrm{L} 1_{0}$ phase) attract interest from both fundamental and applied points of view. A characteristic feature of such transitions is the formation in the ordered phase of peculiar 'polytwinned' structures consisting of arrays of ordered bands separated by the antiphase boundaries (APBs) lying in the (110)-type planes, while the tetragonal axes of antiphase-ordered domains (APDs) in the adjacent bands have 'twin-related' (100)- and (010)-type orientations [1-7]. The transformation A1 $\rightarrow \mathrm{L}_{0}$ includes a number of intermediate stages, including the 'tweed' stage discussed below. These transformations are inherent, in particular, to many alloy systems with outstanding magnetic characteristics, such as $\mathrm{Co}-\mathrm{Pt}, \mathrm{Fe}-\mathrm{Pt}, \mathrm{Fe}-\mathrm{Pd}$, and similar alloys, and studies of their microstructural features, for example, the properties and evolution of APBs, are interesting in

1 Current address: Ames Laboratory, Ames, IA 50011, USA. 
relation to applications of these systems in various magnetic devices for which the structure and the distribution of APBs can be very important [2-4].

The physical reason for the formation of twinned structures has been discussed by a number of authors [8-11], and it is explained by the elimination of the volume-dependent part of elastic energy for such structures. However, theoretical treatments of the kinetics of $\mathrm{A} 1 \rightarrow \mathrm{L} 1_{0}$ transformation seem to be rather scarce as yet. Khachaturyan and co-workers [12] discussed the kinetics of tweed and twin formation using a 2D model in a square lattice with a number of simplifying approximations: a mean-field-type kinetic equation; a phenomenological description of the interaction between elastic strains and local order parameters; an isotropic elasticity; an unrealistic interatomic interaction model (with the nearest-neighbour interaction being weaker by an order of magnitude than more distant interactions); etc. In spite of all these assumptions, some features of the evolution found by Khachaturyan and co-workers [12] agree qualitatively with experimental observations [2-4]. This may illustrate a low sensitivity of these features to the real structure and interactions in an alloy. However, such an oversimplified approach is evidently insufficient for studying the details of the evolution and their dependence on the characteristics of an alloy, such as the type of interatomic interaction, concentration, temperature, etc, which seem to be of most interest for both applications and physical studies of the problem.

In this work we investigate the kinetics of the $\mathrm{A} 1 \rightarrow \mathrm{L} 1_{0}$ transition using the microscopical master equation approach and the kinetic cluster field method [13,14]. Earlier this method was used to study $\mathrm{A} 1 \rightarrow \mathrm{L1}_{2}$-type transformations [15] as well as early stages of the $\mathrm{A} 1 \rightarrow \mathrm{L} 1_{0}$ transition when the deformational interaction $H_{d}$ due to the tetragonal distortion of the $\mathrm{L} 1_{0}$ phase is still insignificant as regards the evolution [16]. Here we consider all stages of this transition, including the tweed and twin stages when the interaction $H_{d}$ becomes important. To this end we first derive a microscopical model for $H_{d}$ which generalizes the analogous model of Khachaturyan for dilute alloys [10] to the physically interesting case of concentrated alloys. Then we employ the kinetic cluster field method to simulate $\mathrm{A} 1 \rightarrow \mathrm{L} 1_{0}$ transformation in the presence of deformational interaction $H_{d}$ for a number of alloy models with both short-range and extended-range chemical interactions at different temperatures, concentrations, and tetragonal deformations. The simulations reveal a number of interesting microstructural features, many of them agreeing well with experimental observations [2-4]. We observe, in particular, a peculiar phenomenon of an interaction-dependent alignment of orientations of APBs within twin bands which was earlier discussed phenomenologically [11]. The simulations also show that the type of microstructural evolution strongly depends on the interaction type as well as on the concentration $c$ and temperature $T$. In particular, drastic, phase-transition-like changes in morphology of APBs within twin bands can occur with variation of $c$ or $T$ in the short-range-interaction systems.

The paper is organized as follows. In section 2 we derive a microscopical expression for the deformational interaction $H_{d}$ in concentrated alloys. In section 3 we describe our methods of simulation of the $\mathrm{A} 1 \rightarrow \mathrm{L}_{0}$ transition which are similar to those used earlier $[15,16]$. In section 4 we investigate the transformation kinetics for the alloy systems with an extended or intermediate interaction range, and in section 5, that for the short-range-interaction systems. Our main conclusions are summarized in section 6.

\section{Model for deformational interaction in concentrated alloys}

\subsection{Hamiltonian}

We consider a binary substitutional alloy $\mathrm{A}_{c} \mathrm{~B}_{1-c}$. Various distributions of atoms over lattice sites $i$ are described using the sets of occupation numbers $\left\{n_{i}\right\}$ where the operator $n_{i}=n_{\mathrm{A} i}$ 
is unity when the site $i$ is occupied by atom A and zero otherwise. The effective Hamiltonian $H_{\text {eff }}$ describing the energy of these distributions has the form

$$
H_{\mathrm{eff}}=\sum_{i>j} v_{i j} n_{i} n_{j}+\sum_{i>j>k} v_{i j k} n_{i} n_{j} n_{k}+\cdots
$$

where $v_{i \ldots j}$ are effective interactions.

The interactions $v_{i \ldots j}$ include the 'chemical' contributions $v_{i \ldots j}^{c}$ which describe the energy changes under the replacement of some atoms A by atoms B in the rigid lattice, and the 'deformational' interactions $v_{i \ldots j}^{d}$ related to the difference in lattice deformation under such a replacement. A microscopical model for $v^{d}$ in dilute alloys was suggested by Khachaturyan [10]. The deformational interaction in concentrated alloys can lead to some new effects that are absent in the dilute alloys, in particular, to the lattice symmetry changes under phase transformations, such as the tetragonal distortion under $\mathrm{L} 1_{0}$ ordering. Earlier these effects were treated only phenomenologically [12]. Below we describe a microscopical model for calculations of $v^{d}$ which generalizes Khachaturyan's approach [10] to the case of concentrated alloys.

Let us denote the position of site $k$ in the disordered 'averaged' crystal as $\boldsymbol{r}_{k}$. Because of the randomness of a real disordered or partially ordered alloy the actual atomic position (averaged over thermal vibrations) is not $\boldsymbol{r}_{k}$ but $\boldsymbol{r}_{k}+\boldsymbol{u}_{k}$ where $\boldsymbol{u}_{k}$ is the 'static displacement'. Supposing this displacement to be small, we can expand the 'adiabatic' (averaged over rapid phonon motion) alloy energy $H=H\left\{n_{i}, \boldsymbol{u}_{k}\right\}$ to second order in $\boldsymbol{u}_{k}$ :

$$
H=H_{c}\left\{n_{i}\right\}-\sum_{k} u_{\alpha k} F^{\alpha k}+\frac{1}{2} \sum_{k, l} u_{\alpha k} u_{\beta l} A_{\alpha k, \beta l}
$$

where $\alpha$ and $\beta$ are Cartesian indices and the summation over repeated Greek indices is implied here and below. The term $H_{c}\left\{n_{i}\right\}$ in (2) describes interactions in the undistorted average crystal lattice, i.e. chemical interactions $v_{i \ldots j}^{c}$ mentioned above; $F^{\alpha k}$ can be called the generalized Kanzaki force; and $A_{\alpha k, \beta l}$ is the force constant matrix. Both quantities $F^{\alpha k}$ and $A_{\alpha k, \beta l}$ are certain functions of occupation numbers $n_{i}$, and their evaluation needs some further approximations.

Below, we consider ordering phase transitions at the fixed mean concentration $c$. Changes of elastic constants and phonon spectra under such transitions are usually small [18]. Therefore, the force constant matrix $A_{\alpha k, \beta l}$ can be reasonably well approximated with the simple 'average crystal' approximation: $A_{\alpha k, \beta l}\left\{n_{i}\right\} \rightarrow A_{\alpha k, \beta l}\{c\} \equiv \bar{A}_{\alpha k, \beta l}$. To approximate the Kanzaki force $F^{\alpha k}$ we first formally write it as a series in the occupation numbers $n_{i}$ :

$$
F^{\alpha k}\left\{n_{i}\right\}=F_{0}^{\alpha k}+\sum_{i} F_{1}^{\alpha k, i} n_{i}+\sum_{i>j} F_{2}^{\alpha k, i j} n_{i} n_{j}+\cdots .
$$

Equilibrium values of displacements $\boldsymbol{u}_{k}=\boldsymbol{u}_{k}^{e}\left\{n_{i}\right\}$ at the given distribution $\left\{n_{i}\right\}$ are determined by the minimization of energy (2) over $\boldsymbol{u}_{k}$, and the constant $F_{0}^{\alpha k}$ in (3) affects only the reference point $\boldsymbol{u}_{k}^{e}\{0\}$ in the function $\boldsymbol{u}_{k}^{e}\left\{n_{i}\right\}$. This constant can be determined, for example, from the condition of vanishing of mean static displacements in the averaged crystal at some $c=c_{0}$, which implies the relation $\left\langle F^{\alpha k}\left\{n_{i}\right\}\right\rangle_{c=c_{0}}=0$ where the symbol $\langle\cdots\rangle$ means the statistical averaging over an alloy. The constants $F_{0}^{\alpha k}$ are insignificant in what follows, and below they are omitted to simplify formulae.

In writing an explicit expression for the contribution $H_{K}$ (to be called for brevity the 'Kanzaki term') of the occupation-dependent Kanzaki forces in energy (2), one should consider that due to the translation invariance it can include only differences of displacements $\left(\boldsymbol{u}_{k}-\boldsymbol{u}_{i}\right)$, $\left(\boldsymbol{u}_{k}-\boldsymbol{u}_{j}\right)$, etc. Therefore, this term should have the form

$$
H_{K}=\sum_{k, i}\left(\boldsymbol{u}_{i}-\boldsymbol{u}_{k}\right) \boldsymbol{f}_{1}^{k, i} n_{i}+\sum_{k, i j}\left(\boldsymbol{u}_{i}-\boldsymbol{u}_{k}\right) \boldsymbol{f}_{2}^{k, i j} n_{i} n_{j}+\cdots
$$


where $f_{m}^{k, i_{1} \ldots i_{m}} \equiv \boldsymbol{f}_{m}^{k}$ are some parameters describing the interaction of lattice deformations with site occupations.

Representation (4) for $H_{K}$ as a sum of contributions of $m$-site 'clusters' proportional to products $n_{i_{1}} \cdots n_{i_{m}}$ is analogous to similar cluster expansions for the 'chemical' Hamiltonian $H_{c}\left\{n_{i}\right\}$ in (2). These expansions have been widely discussed, in particular, in connection with first-principles calculations of chemical interactions $v_{i_{1} \ldots i_{m}}^{c} \equiv v^{c, m}$; see e.g. [19]. The calculations have shown that the values of the $m$-site interactions $v^{c, m}$ in most alloys rapidly decrease with increase of $m$, and the pairwise interaction $v^{c, 2}$ is usually dominant (though the possible importance of many-particle interactions related, in particular, to the charge-transfer effects is also debated [17]). It is natural to expect a similar rapid convergence to also be typical for the expansion (4). Therefore, below, we omit many-site interactions $f_{m}^{k}$ with $m>2$ in equation (4). At the same time, in estimates of parameters $f_{m}^{k}$ for real alloys below, we combine some model assumptions about $f_{m}^{k}$ with use of available experimental data on the variations of lattice deformations with concentration and orderings, and such estimates may also implicitly include the contributions of many-site interactions $f_{m}^{k}$.

For what follows it is convenient to proceed from the functions $\boldsymbol{u}_{k}=\boldsymbol{u}\left(\boldsymbol{r}_{k}\right), n_{i}=n\left(\boldsymbol{r}_{i}\right)$, $\boldsymbol{f}_{1}^{k, i}=\boldsymbol{f}_{1}\left(\boldsymbol{r}_{k}-\boldsymbol{r}_{i}\right), \boldsymbol{f}_{2}^{k, i j}=\boldsymbol{f}_{2}\left(\boldsymbol{r}_{k}-\boldsymbol{r}_{i}, \boldsymbol{r}_{j}-\boldsymbol{r}_{i}\right)$, and $\bar{A}_{\alpha k, \beta l}=\bar{A}_{\alpha \beta}\left(\boldsymbol{r}_{k}-\boldsymbol{r}_{l}\right)$ in equations (2) and (4) to their Fourier components in the average crystal lattice. Then the energy (2) takes the form

$$
H=H_{c}\left\{n_{i}\right\}+\frac{1}{N} \sum_{k} \boldsymbol{u}_{-k}\left(n_{k} \boldsymbol{f}_{1 k}+\sum_{\boldsymbol{R}} \sigma_{k}^{R} \boldsymbol{f}_{2 k}^{R}\right)+\frac{1}{2 N} \sum_{k} u_{-k}^{\alpha} \bar{A}_{k}^{\alpha \beta} u_{k}^{\beta} .
$$

Here $N$ is the total number of crystal cells, the summation over $\boldsymbol{k}$ is within the Brillouin zone of the averaged crystal, and we use the following notation:

$$
\begin{array}{rlrl}
\boldsymbol{u}_{k} & =\sum_{r} \boldsymbol{u}(\boldsymbol{r}) \mathrm{e}^{-\mathrm{i} k \cdot \boldsymbol{r}} \quad n_{k}=\sum_{r} n(\boldsymbol{r}) \mathrm{e}^{-\mathrm{i} k \cdot \boldsymbol{r}} \quad \sigma_{k}^{\boldsymbol{R}}=\sum_{r} n(\boldsymbol{r}) n(\boldsymbol{r}+\boldsymbol{R}) \mathrm{e}^{-\mathrm{i} k \cdot \boldsymbol{r}} \\
\boldsymbol{f}_{1 k} & =\sum_{r} \boldsymbol{f}_{1}(\boldsymbol{r})\left(1-\mathrm{e}^{-\mathrm{i} k \cdot \boldsymbol{r}}\right) & \boldsymbol{f}_{2 k}^{\boldsymbol{R}}=\sum_{r} \boldsymbol{f}_{2}(\boldsymbol{r}, \boldsymbol{R})\left(1-\mathrm{e}^{-\mathrm{i} k \cdot \boldsymbol{r}}\right) \\
\bar{A}_{k}^{\alpha \beta} & =\sum_{r} \bar{A}_{\alpha \beta}(\boldsymbol{r}) \mathrm{e}^{-\mathrm{i} k \cdot \boldsymbol{r}} .
\end{array}
$$

If one adopts a commonly used model of 'central' Kanzaki forces in which forces $f_{1}^{k, i}$ and $\boldsymbol{f}_{2}^{k, i j}$ in (4) are supposed to be proportional to the vector $\boldsymbol{r}_{k i}=\left(\boldsymbol{r}_{k}-\boldsymbol{r}_{i}\right)$, the vector functions $\boldsymbol{f}_{1 k}$ and $\boldsymbol{f}_{2 k}$ in (6) can be expressed via two scalar functions, $\varphi_{1}$ and $\varphi_{2}$ :

$$
\boldsymbol{f}_{1 k}=\sum_{r} \boldsymbol{r} \varphi_{1}(r)\left(1-\mathrm{e}^{-\mathrm{i} k \cdot \boldsymbol{r}}\right) \quad \boldsymbol{f}_{2 \boldsymbol{k}}^{\boldsymbol{R}}=\sum_{r} \boldsymbol{r} \varphi_{2}(\boldsymbol{r}, \boldsymbol{R})\left(1-\mathrm{e}^{-\mathrm{i} k \cdot \boldsymbol{r}}\right) .
$$

\subsection{Elastic energy for disordered and ordered phases}

The functions $\varphi_{1}$ and $\varphi_{2}$ in (7) determine the dependence of equilibrium lattice parameters on concentration or ordering. To show this, we first note that the homogeneous deformation $\bar{u}_{\alpha \beta}$ is described in terms of Fourier components $\boldsymbol{u}_{k}$ with small $k \rightarrow 0$, while functions $\boldsymbol{f}_{1 k}$ and $\boldsymbol{f}_{2 \boldsymbol{k}}$ in equations (5) and (6) at small $\boldsymbol{k}$ are linear in $\boldsymbol{k}$. Thus the contribution of homogeneous deformations to the Kanzaki term in (5) is proportional to Fourier components $u_{k}^{\alpha \beta}$ of the elastic strain $u_{\alpha \beta}=\left(\partial u_{\alpha} / \partial x_{\beta}+\partial u_{\beta} / \partial x_{\alpha}\right) / 2$ for $\boldsymbol{k} \rightarrow 0$ and, according to first equation (6), these components are related to $\bar{u}_{\alpha \beta}$ as

$$
\left.u_{k}^{\alpha \beta}\right|_{k \rightarrow 0}=\left.\mathrm{i}\left(k_{\beta} u_{k}^{\alpha}+k_{\alpha} u_{k}^{\beta}\right)\right|_{k \rightarrow 0}=N \bar{u}_{\alpha \beta} .
$$

At small $k$ the force constant matrix $\bar{A}_{k}^{\alpha \beta}$ in (5) is bilinear in $k$, and the last term of (5) corresponds to the standard expression for the elastic energy bilinear in $\bar{u}_{\alpha \beta}$ and linear in 
the elastic constants $c_{\alpha \beta \gamma \delta}$; see e.g. [10]. Therefore, the total contribution of terms with the homogeneous elastic strain $\bar{u}_{\alpha \beta}$ to energy (5) (to be called 'the elastic strain energy' $E_{\mathrm{el}}$ ) can be written as

$$
E_{\mathrm{el}}=-\bar{u}_{\alpha \beta}\left(A_{1}^{\alpha \beta} n_{0}+\sum_{\boldsymbol{R}} A_{2 \boldsymbol{R}}^{\alpha \beta} \sigma_{0}^{\boldsymbol{R}}\right)+\frac{1}{2} N \Omega c_{\alpha \beta \gamma \delta} \bar{u}_{\alpha \beta} \bar{u}_{\gamma \delta} .
$$

Here $\Omega$ is the volume per atom in the average crystal; quantities $A_{1}^{\alpha \beta}$ and $A_{2 R}^{\alpha \beta}$ are expressed via functions $\varphi_{1}$ and $\varphi_{2}$ in (7) as

$$
A_{1}^{\alpha \beta}=\sum_{r} x_{\alpha} x_{\beta} \varphi_{1}(\boldsymbol{r}) \quad A_{2 \boldsymbol{R}}^{\alpha \beta}=\sum_{r} x_{\alpha} x_{\beta} \varphi_{2}(\boldsymbol{r}, \boldsymbol{R})
$$

where $x_{\alpha}$ is the Cartesian component of vector $r=\left(x_{1}, x_{2}, x_{3}\right)$; and $n_{0}$ or $\sigma_{0}^{R}$ is the Fourier component, $n_{k}$ or $\sigma_{k}^{R}$, at $k=0$. According to equation (6), the operator $n_{0}$ or $\sigma_{0}^{R}$ is the sum of a macroscopically large number $N$ of similar terms. Thus within the statistical accuracy each of these operators can be replaced by its average value:

$$
n_{0}=N\langle n(\boldsymbol{r})\rangle=N c \quad \sigma_{0}^{\boldsymbol{R}}=N\langle n(\boldsymbol{r}) n(\boldsymbol{r}+\boldsymbol{R})\rangle .
$$

The last average in (11) can be expressed via mean occupations of sites and their correlators. In an ordered alloy there exist several non-equivalent sublattices $s$ with the lattice vectors $\boldsymbol{r}_{s}$ and mean occupations $c_{s}=\left\langle n\left(\boldsymbol{r}_{s}\right)\right\rangle$, and so the last average in (11) includes averaging over all sublattices $s$ :

$$
\langle n(\boldsymbol{r}) n(\boldsymbol{r}+\boldsymbol{R})\rangle=\sum_{s} v_{s}\left(c_{s} c_{s \boldsymbol{R}}+K_{s \boldsymbol{R}}\right)
$$

Here $c_{s} \boldsymbol{R}$ is the mean occupation $\langle n(\boldsymbol{r})\rangle$ for $\boldsymbol{r}=\boldsymbol{r}_{s}+\boldsymbol{R} ; v_{s}=N_{s} / N$ is the relative number of sites in the sublattice $s$; and $K_{s}$ is the correlator of occupations of sites located at $r=r_{s}$ and at $\boldsymbol{r}=\boldsymbol{r}_{s}+\boldsymbol{R}$ :

$$
K_{s \boldsymbol{R}}=\left\langle\left[n\left(\boldsymbol{r}_{s}\right)-c_{s}\right]\left[n\left(\boldsymbol{r}_{s}+\boldsymbol{R}\right)-c_{s \boldsymbol{R}}\right]\right\rangle .
$$

In a disordered alloy all sites are equivalent. Thus, $c_{s}=c_{s R}=c ; v_{s}=1$; and both index $s$ and the summation over $s$ in (12) are omitted.

Using equations (11) and (12) one can rewrite the elastic strain energy (9) as

$E_{\mathrm{el}}=-N \bar{u}_{\alpha \beta}\left[A_{1}^{\alpha \beta} c+\sum_{\boldsymbol{R}} \sum_{s} v_{s}\left(c_{s} c_{s \boldsymbol{R}}+K_{s \boldsymbol{R}}\right) A_{2 \boldsymbol{R}}^{\alpha \beta}\right]+\frac{1}{2} N \Omega c_{\alpha \beta \gamma \delta} \bar{u}_{\alpha \beta} \bar{u}_{\gamma \delta}$.

The correlator $K_{s R}$ in equation (14) can be calculated using that or another method of statistical theory. However, for most alloy systems of practical interest, in particular, at $c$ - and $T$-values not close to the thermodynamic instability points $T_{s}$, the correlators $K_{s R}$ are small and can be neglected. Then equation (14) is simplified:

$$
E_{\mathrm{el}}=-\bar{u}_{\alpha \beta}\left[N A_{1}^{\alpha \beta} c+\sum_{\boldsymbol{r}, \boldsymbol{R}} c(\boldsymbol{r}) c(\boldsymbol{r}+\boldsymbol{R}) A_{2 \boldsymbol{R}}^{\alpha \beta}\right]+\frac{1}{2} N \Omega c_{\alpha \beta \gamma \delta} \bar{u}_{\alpha \beta} \bar{u}_{\gamma \delta} .
$$

Equilibrium values of $\bar{u}_{\alpha \beta}$ in the absence of applied stress are determined by the minimization of energy $E_{\mathrm{el}}$ with respect to $\bar{u}_{\alpha \beta}$ which gives

$$
\Omega c_{\alpha \beta \gamma \delta} \bar{u}_{\gamma \delta}=A_{1}^{\alpha \beta} c+\frac{1}{N} \sum_{r, \boldsymbol{R}} c(\boldsymbol{r}) c(\boldsymbol{r}+\boldsymbol{R}) A_{2 \boldsymbol{R}}^{\alpha \beta} .
$$

Equation (16) enables one to express the equilibrium strain $\bar{u}_{\alpha \beta}$ via the concentration, order parameters, and the interaction parameters $A_{1}^{\alpha \beta}$ and $A_{2 R}^{\alpha \beta}$, and it can also be used to estimate these interaction parameters from experimental data on $\bar{u}_{\alpha \beta}(c, T)$. 
Let us consider equations (15) and (16) in particular cases. For a disordered phase with $c(\boldsymbol{r})=c$, equation (16) takes the form

$$
\Omega c_{\alpha \beta \gamma \delta} \bar{u}_{\gamma \delta}=A_{1}^{\alpha \beta} c+A_{2}^{\alpha \beta} c^{2}
$$

where $A_{2}^{\alpha \beta}=\sum_{R} A_{2 R}^{\alpha \beta}$. If the disordered phase has a cubic symmetry (as for the FCC or BCC alloys), quantities $A_{1}^{\alpha \beta}$ and $A_{2}^{\alpha \beta}$ are proportional to the Kronecker symbol $\delta_{\alpha \beta}$, and equation (17) determines the concentrational dilatation $u(c)=\bar{u}_{\alpha \alpha}(c)-u_{\alpha \alpha}(0)$ :

$$
u(c)=\left(A_{1} c+A_{2} c^{2}\right) / \Omega B .
$$

Here $B=\left(c_{11}+2 c_{12}\right) / 3$ is the bulk modulus; $c_{i j}$ are the elastic constants in Voigt's notation; and coefficients $A_{1}$ and $A_{2}$ are expressed via functions $\varphi_{1}$ and $\varphi_{2}$ in (7), (10):

$$
A_{1}=\sum_{r} \varphi_{1}(\boldsymbol{r}) r^{2} / 3 \quad A_{2}=\sum_{r, \boldsymbol{R}} \varphi_{2}(\boldsymbol{r}, \boldsymbol{R}) r^{2} / 3
$$

The linear-in- $c$ term in (18) corresponds to the Vegard law while the term with $A_{2}$ describes the non-linear deviations from this law. Such deviations were observed for many alloys, and these data can be used to estimate $A_{2}$-values, but in these estimates one should also take into consideration a possible concentration dependence of the bulk modulus $B$.

For the ordered phase, the mean occupation $c(\boldsymbol{r})$ can be written as a superposition of concentration waves corresponding to certain superstructure vectors $\boldsymbol{k}_{p}$ [10]:

$$
c(\boldsymbol{r})=c+\frac{1}{2} \sum_{p}\left[\eta_{p} \exp \left(\mathrm{i} \boldsymbol{k}_{p} \cdot \boldsymbol{r}\right)+\eta_{p}^{*} \exp \left(-\mathrm{i} \boldsymbol{k}_{p} \cdot \boldsymbol{r}\right)\right]
$$

and amplitudes $\eta_{p}$ can be considered as order parameters. After the substitution of expressions (20) for $c(\boldsymbol{r})$ and $c(\boldsymbol{r}+\boldsymbol{R})$ in equation (15) the linear-in- $\eta_{p}$ terms vanish due to the crystal symmetry, and the first term of (15) becomes the sum of the ordering-independent term and the term bilinear in the order parameters:

$$
E_{\mathrm{el}}=-N \bar{u}_{\alpha \beta}\left(A_{1}^{\alpha \beta} c+A_{2}^{\alpha \beta} c^{2}+\sum_{p} q_{\alpha \beta p p}\left|\eta_{p}\right|^{2}\right)+\frac{1}{2} N \Omega c_{\alpha \beta \gamma \delta} \bar{u}_{\alpha \beta} \bar{u}_{\gamma \delta} .
$$

Here quantities $q_{\alpha \beta p p}$ have a different form in the cases (a) when the superstructure vector $\boldsymbol{k}_{p}$ is half of some reciprocal-lattice vector $\boldsymbol{g}$ and thus both the order parameter $\eta_{p}$ and all factors $\exp \left(\boldsymbol{k}_{p} \cdot \boldsymbol{r}\right)$ in (20) are real, and (b) when $\boldsymbol{k}_{p} \neq \boldsymbol{g} / 2$ :

$$
\begin{array}{rlrl}
\text { (a) } & \boldsymbol{k}_{p}=\boldsymbol{g} / 2: & q_{\alpha \beta p p} & =\sum_{\boldsymbol{r}, \boldsymbol{R}} x_{\alpha} x_{\beta} \varphi_{2}(\boldsymbol{r}, \boldsymbol{R}) \exp \left(\mathrm{i} \boldsymbol{k}_{p} \cdot \boldsymbol{r}\right) \\
\text { (b) } & \boldsymbol{k}_{p} \neq \boldsymbol{g} / 2: & q_{\alpha \beta p p}=\frac{1}{2} \sum_{\boldsymbol{r}, \boldsymbol{R}} x_{\alpha} x_{\beta} \varphi_{2}(\boldsymbol{r}, \boldsymbol{R}) \cos \left(\boldsymbol{k}_{p} \cdot \boldsymbol{r}\right) .
\end{array}
$$

The coefficients $q_{\alpha \beta p p}$ in (21) (to be called the 'striction' coefficients, in analogy with the terminology used in the ferroelectricity or magnetism theory) are commonly used in phenomenological theories of lattice distortions under orderings [8-12]. Equations (22), (23), and (10) provide the microscopic expression for these coefficients via the function $\varphi_{2}$ describing non-pairwise Kanzaki forces in equations (5)-(7).

\subsection{Striction effects in the $\mathrm{LI}_{2}$ and $\mathrm{LI}_{0}$ phases}

Let us apply equations (20)-(22) to the case of $\mathrm{L}_{0}$ or $\mathrm{L}_{2}$ ordering in $\mathrm{FCC}$ alloys which are described by means of three real order parameters $\eta_{\alpha}[10,15]$. Equation (20) here take the form

$$
c(\boldsymbol{r})=c+\eta_{1} \exp \left(\mathrm{i}_{1} \cdot \boldsymbol{r}\right)+\eta_{2} \exp \left(\mathrm{i}_{2} \cdot \boldsymbol{r}\right)+\eta_{3} \exp \left(\mathrm{i} \boldsymbol{k}_{3} \cdot \boldsymbol{r}\right)
$$


where $\boldsymbol{k}_{\alpha}=\boldsymbol{g}_{\alpha} / 2$ is the superstructure vector corresponding to $\eta_{\alpha}$ :

$$
\boldsymbol{k}_{1}=[100] 2 \pi / a \quad \boldsymbol{k}_{2}=[010] 2 \pi / a \quad \boldsymbol{k}_{3}=[001] 2 \pi / a .
$$

In the cubic $\mathrm{L} 1_{2}$ structure one has $\left|\eta_{1}\right|=\left|\eta_{2}\right|=\left|\eta_{3}\right|, \eta_{1} \eta_{2} \eta_{3}>0$, and four types of ordered domain are possible. In the $\mathrm{L} 1_{0}$-ordered structure with the tetragonal axis $\alpha$, a single parameter $\eta_{\alpha}$ is present which is either positive or negative, and so six types of ordered domain are possible.

The striction coefficients for $\mathrm{L} 1_{0}$ or $\mathrm{L}_{2}$ ordering are determined by equation (22). Due to the cubic symmetry of the 'average' FCC crystal, there are only two different striction coefficients, $q_{1111}$ and $q_{1122}$ (and those obtained from them by the cubic symmetry operations), which for brevity will be denoted as $q_{11}$ and $q_{12}$, respectively:

$$
q_{11}=\sum_{\boldsymbol{r}, \boldsymbol{R}} x_{1}^{2} \varphi_{2}(\boldsymbol{r}, \boldsymbol{R}) \exp \left(\mathrm{i} \boldsymbol{k}_{1} \cdot \boldsymbol{R}\right) \quad q_{12}=\sum_{\boldsymbol{r}, \boldsymbol{R}} x_{1}^{2} \varphi_{2}(\boldsymbol{r}, \boldsymbol{R}) \exp \left(\mathrm{i} \boldsymbol{k}_{2} \cdot \boldsymbol{R}\right) .
$$

The variation of the elastic constants $c_{\alpha \beta \gamma \delta}$ with ordering is usually small [18], and for simplicity it will be neglected. Then minimizing energy (21) with respect to $\bar{u}_{\alpha \beta}$ we obtain the expressions for the lattice deformations induced by ordering (24):

$$
\begin{aligned}
\bar{u} & =q_{+}\left(\eta_{1}^{2}+\eta_{2}^{2}+\eta_{3}^{2}\right) / \Omega c_{+} \quad \bar{\varepsilon}=q_{-}\left[\eta_{1}^{2}-\left(\eta_{2}^{2}+\eta_{3}^{2}\right) / 2\right] / \Omega c_{-} \\
\zeta & =q_{-}\left(\eta_{2}^{2}-\eta_{3}^{2}\right) / \Omega c_{-} .
\end{aligned}
$$

Here $\bar{u}=\bar{u}_{11}+\bar{u}_{22}+\bar{u}_{33}$ describes the volume change; $\bar{\varepsilon}=\bar{u}_{11}-\left(\bar{u}_{22}+\bar{u}_{33}\right) / 2$ is the tetragonal distortion; $\zeta=\bar{u}_{22}-\bar{u}_{33}$ is the shear deformation; and $q_{ \pm}$or $c_{ \pm}$are linear combinations of striction or elastic constants:

$q_{-}=q_{11}-q_{12} \quad c_{-}=c_{11}-c_{12} \quad q_{+}=q_{11}+2 q_{12} \quad c_{+}=c_{11}+2 c_{12}$.

For the $\mathrm{L}_{2}$ ordering, the values $\left|\eta_{1}\right|=\left|\eta_{2}\right|=\left|\eta_{3}\right|=\eta$ are the same, so just the volume striction $\bar{u}=3 q_{+} \eta^{2} / \Omega c_{+}$is present, while in the $\mathrm{L} 1_{0}$-ordered domain with $\eta_{2}=\eta_{3}=0$ one has both the volume and the tetragonal striction:

$$
\bar{u}=q_{+} \eta_{1}^{2} / \Omega c_{+} \quad \bar{\varepsilon}=q_{-} \eta_{1}^{2} / \Omega c_{-} .
$$

Therefore, using experimental data on the lattice distortions and order parameters under $\mathrm{L} 1_{2}$ and $\mathrm{L}_{0}$ orderings, one can estimate the striction coefficients $q_{11}$ and $q_{12}$ and thus the nonpairwise Kanzaki interaction $\varphi_{2}$ in equations (26).

\subsection{The short-range Kanzaki force model}

Below we suppose for simplicity the interaction $\varphi_{2}(\boldsymbol{r}, \boldsymbol{R})$ to be short ranged, i.e. significant only when each of three relative distances $r, R$, and $|\boldsymbol{r}-\boldsymbol{R}|$ does not exceed the nearest-neighbour distance $\rho=a / \sqrt{2}$. Then this function can be written as

$$
\varphi_{2}(\boldsymbol{r}, \boldsymbol{R})=\delta_{r, \rho} \delta_{R, \rho}\left(\varphi_{a} \delta_{|r-\boldsymbol{R}|, 0}+\varphi_{b} \delta_{|\boldsymbol{r}-\boldsymbol{R}|, \rho}\right)
$$

where $\delta_{r, \rho}$ is the Kronecker symbol equal to unity when $r=\rho$ and zero otherwise while $\varphi_{a}$ and $\varphi_{b}$ are the interaction parameters. The assumption (30) is analogous to that used by Khachaturyan [10] for the pairwise Kanzaki interaction $\varphi_{1}(\boldsymbol{r})$ in (7):

$$
\varphi_{1}(r)=\varphi_{1} \delta_{r, \rho}
$$

where the constant $\varphi_{1}$ is estimated from experimental data on concentrational dilatation. First-principles estimates of lattice distortions in dilute alloys [20] seem to imply that the assumption (31) yields the correct order of magnitude of $\varphi_{1}(\boldsymbol{r})$. Therefore, the analogous assumption (30) for $\varphi_{2}(\boldsymbol{r}, \boldsymbol{R})$ should be reasonable, too. Let us also note that the term with $\varphi_{a}$ in (30) corresponds to the two-site interaction between the occupations $n_{i}$ and $n_{j}$ of neighbouring sites $i$ and $j$ and their relative dilatation $\left(\boldsymbol{u}_{i}-\boldsymbol{u}_{j}\right)$, while the term with 
$\varphi_{b}$ describes the three-site interaction between the occupations $n_{i}$ and $n_{j}$ and the relative displacement $\left(\boldsymbol{u}_{k}-\boldsymbol{u}_{i}\right)$ of the third adjacent site $k$.

Substituting equation (30) into (26), we obtain the explicit expression for coefficients $q_{i k}$ via parameters $\varphi_{a}$ and $\varphi_{b}$ in (30):

$$
q_{11}=-2 a^{2} \varphi_{a} \quad q_{12}=-4 a^{2} \varphi_{b}
$$

The coefficient $f_{1 k}$ in equations (5) and (6) for model (31) has the form [10]

$$
\boldsymbol{f}_{1 k}=4 \varphi_{1} \mathrm{i} \sum_{\alpha=1}^{3} \boldsymbol{a}_{\alpha} \sin \left(\boldsymbol{k} \cdot \boldsymbol{a}_{\alpha}\right) \sum_{\beta \neq \alpha} \cos \left(\boldsymbol{k} \cdot \boldsymbol{a}_{\beta}\right)
$$

where $\boldsymbol{a}_{\alpha}$ is $\boldsymbol{e}_{\alpha} a / 2$ and $\boldsymbol{e}_{\alpha}$ is the unit vector along the main crystal axis $\alpha$. The function $\boldsymbol{f}_{2 k}^{R}$ in (5), (6) for model (30) is the sum of two terms:

$$
f_{2 k}^{R}=f_{a k}^{R}+f_{b k}^{R}
$$

Here, $\boldsymbol{f}_{a \boldsymbol{k}}^{\boldsymbol{R}}$ is $\varphi_{a} \delta_{R, \rho} \boldsymbol{R}\left(1-\mathrm{e}^{-\mathrm{i} k \cdot \boldsymbol{R}}\right)$, while the function $\boldsymbol{f}_{b \boldsymbol{k}}^{\boldsymbol{R}}$ for $\boldsymbol{R}$ equal to $\boldsymbol{R}_{\alpha}+\boldsymbol{R}_{\beta}$ (where $\boldsymbol{R}_{\alpha}$ is $\boldsymbol{a}_{\alpha}$ or $\left(-\boldsymbol{a}_{\alpha}\right), \boldsymbol{R}_{\beta}$ is $\boldsymbol{a}_{\beta}$ or $\left(-\boldsymbol{a}_{\beta}\right)$, and $\left.\beta \neq \alpha\right)$ can be written as

$$
\begin{aligned}
\boldsymbol{f}_{b \boldsymbol{k}}^{\boldsymbol{R}}=2 \varphi_{b}[\boldsymbol{R} & +\left(\mathrm{i} \boldsymbol{a}^{\prime} \sin \left(\boldsymbol{k} \cdot \boldsymbol{a}^{\prime}\right)-\boldsymbol{R}_{\alpha} \cos \left(\boldsymbol{k} \cdot \boldsymbol{a}^{\prime}\right)\right) \mathrm{e}^{-\mathrm{i} \boldsymbol{k} \cdot \boldsymbol{R}_{\alpha}} \\
& \left.+\left(\mathrm{i} \boldsymbol{a}^{\prime} \sin \left(\boldsymbol{k} \cdot \boldsymbol{a}^{\prime}\right)-\boldsymbol{R}_{\beta} \cos \left(\boldsymbol{k} \cdot \boldsymbol{a}^{\prime}\right)\right) \mathrm{e}^{-\mathrm{i} \boldsymbol{k} \cdot \boldsymbol{R}_{\beta}}\right]
\end{aligned}
$$

where $\boldsymbol{a}^{\prime}$ is $\left[\boldsymbol{e}_{\alpha} \cdot \boldsymbol{e}_{\beta}\right] a / 2$.

Relations (7), (30)-(35) together with (18) and (32) provide a simplified model for the Kanzaki term $H_{K}$ in equations (4) and (5). This model will be used below in simulations of $\mathrm{A} 1 \rightarrow \mathrm{L} 1_{0}$ transitions. To get an idea of the actual scale of parameters of this model, let us estimate quantities $q_{i k}, \varphi_{a}$, and $\varphi_{b}$ in equations (30) and (32) for the alloys Co-Pt for which detailed data on the lattice distortions under $\mathrm{L}_{2}$ and $\mathrm{L} 1_{0}$ orderings are available $[25,26]$. The volume changes $\bar{u}$ under the $\mathrm{L}_{2}$ ordering in $\mathrm{CoPt}_{3}$ and $\mathrm{L}_{0}$ ordering in CoPt both appear to be very small $[1,25,26]: \bar{u} \lesssim 10^{-3}$. According to equations (27) and (29), this implies the relation $q_{12} \simeq q_{11} / 2$. The value $q_{-}=\left(q_{11}-q_{12}\right)$ for CoPt can be estimated from the second of equations (29) using data from [26] for $\eta_{1}$ and $\bar{\varepsilon}$ at $T=0.84 T_{c}$ : $\eta_{1} \simeq 0.4$; $\varepsilon \simeq-0.04$ (with the thermal expansion effect subtracted); and for the atomic volume: $\Omega=\Omega\left(T_{c}+0\right) \simeq 13.8 \AA^{3}$. Using also for the elastic constant $c_{-}=\left(c_{11}-c_{12}\right)$ its value for FCC platinum, $c_{-} \simeq 0.97$ Mbar [27], we obtain $q_{-} \simeq 2.6 \times 10^{4} \mathrm{~K}$. Combining this with the above-mentioned relation $q_{12} \simeq q_{11} / 2$ and using equation (27), we find $\varphi_{a} \simeq 2 \times 10^{4} \mathrm{~K} / a^{2}$ and $\varphi_{b} \simeq 5 \times 10^{3} \mathrm{~K} / a^{2}$. Let us also note that the ordering-induced elastic energy per atom $\varepsilon_{\mathrm{el}}^{\text {ord }}$ in the CoPt alloy is small: $\varepsilon_{\mathrm{el}}^{\text {ord }} \simeq \Omega c_{-} \bar{\varepsilon}^{2} / 6 \simeq 30 \mathrm{~K}$, which is much less than the $\mathrm{L} 1_{0}$ ordering temperature $T_{c} \simeq 1100 \mathrm{~K}$.

\subsection{Effective deformational interactions}

The equilibrium values of displacements $\boldsymbol{u}_{k}^{e}=\boldsymbol{u}_{\boldsymbol{k}}^{e}\left(n_{i}\right)$ are found by the minimization of energy (5) over $\boldsymbol{u}_{k}$. Substituting these $\boldsymbol{u}_{k}^{e}$ into equation (5), we obtain the effective Hamiltonian $H=H_{c}+H_{d}$ where the deformational interaction $H_{d}$ can be written as

$H_{d}=-\frac{1}{2 N} \sum_{k}\left(n_{-k} f_{1 k}^{*}+\sum_{R} \sigma_{-k}^{R} f_{2 k}^{R *}\right) G_{k}\left(n_{k} f_{1 k}+\sum_{R} \sigma_{k}^{R} f_{2 k}^{R}\right)=H_{d 2}+H_{d 3}+H_{d 4}$

Here the matrix $\boldsymbol{G}_{k}=G_{k}^{\alpha \beta}$ is the inverse of the force constant matrix $\bar{A}_{k}^{\alpha \beta}$, and the matrix product $\boldsymbol{a B c}$ means the sum $a_{\alpha} B_{\alpha \beta} b_{\beta}$. The terms $H_{d 2}, H_{d 3}$, and $H_{d 4}$ in (36) describe the pairwise, three-particle, and four-particle deformational interactions, respectively: 


$$
\begin{aligned}
& H_{d 2}=\frac{1}{2} \sum_{r, \boldsymbol{r}^{\prime}} n(\boldsymbol{r}) \Phi_{2}\left(\boldsymbol{r}-\boldsymbol{r}^{\prime}\right) n\left(\boldsymbol{r}^{\prime}\right) \\
& H_{d 3}=\frac{1}{2} \sum_{\boldsymbol{r}, \boldsymbol{r}^{\prime}} \sum_{\boldsymbol{R}} n(\boldsymbol{r}) \Phi_{3}^{\boldsymbol{R}}\left(\boldsymbol{r}-\boldsymbol{r}^{\prime}\right) n\left(\boldsymbol{r}^{\prime}\right) n\left(\boldsymbol{r}^{\prime}+\boldsymbol{R}\right) \\
& H_{d 4}=\frac{1}{2} \sum_{\boldsymbol{r}, \boldsymbol{r}^{\prime}} \sum_{\boldsymbol{R}, \boldsymbol{R}^{\prime}} n(\boldsymbol{r}) n(\boldsymbol{r}+\boldsymbol{R}) \Phi_{4}^{\boldsymbol{R}, \boldsymbol{R}^{\prime}}\left(\boldsymbol{r}-\boldsymbol{r}^{\prime}\right) n\left(\boldsymbol{r}^{\prime}\right) n\left(\boldsymbol{r}^{\prime}+\boldsymbol{R}^{\prime}\right)
\end{aligned}
$$

where the potential $\Phi_{2}, \Phi_{3}^{R}$, or $\Phi_{4}^{R, R^{\prime}}$ is given by the expression

$$
\begin{aligned}
& \left\{\Phi_{2}(\boldsymbol{r}) ; \Phi_{3}^{R}(\boldsymbol{r}) ; \Phi_{4}^{R, R^{\prime}}(\boldsymbol{r})\right\} \\
& \quad=-\frac{1}{N} \sum_{k} \mathrm{e}^{\mathrm{i} k \cdot r}\left\{f_{1 k}^{*} G_{k} f_{1 k} ; f_{1 k}^{*} G_{k} f_{2 k}^{R}+f_{2 k}^{R *} G_{k} f_{1 k} ; f_{2 k}^{R *} G_{k} f_{2 k}^{R^{\prime}}\right\} .
\end{aligned}
$$

As the matrix $\boldsymbol{G}_{\boldsymbol{k}}$ in (39) at small $k$ includes the well-known 'elastic singularity' [10]: $\boldsymbol{G}_{k} \sim 1 / k^{2}$, each of the terms $H_{d 2}, H_{d 3}$, and $H_{d 4}$ in (36) includes the long-ranged elastic interaction. The formation of twinned structures discussed below is determined by the fourparticle interaction $H_{d 4}$. The rest of the deformational interactions, $H_{d 2}$ and $H_{d 3}$, for the single-phase $\mathrm{L}_{0}$ ordering under consideration, lead just to some quantitative renormalizations of chemical interaction $H_{c}$ in (5) which are usually small and insignificant. Therefore, below, we retain in the deformational interaction (36) only the last term $H_{d 4}$. Let us also note that each term in the sum (38) for $H_{d 4}$ at fixed $\boldsymbol{r}$ and $\boldsymbol{r}^{\prime}$ has the order of magnitude of the above-mentioned ordering-induced elastic energy $\varepsilon_{\mathrm{el}}^{\text {ord }}$ which is usually small. Thus the interaction $H_{d 4}$ can be significant only because of 'coherent' contributions of many sites $\boldsymbol{r}$ and $\boldsymbol{r}^{\prime}$ due to the long-ranged elastic interaction. Therefore, local fluctuations of occupations $n(\boldsymbol{r})$ in the interaction $H_{d 4}$ are insignificant, and it can be treated in the 'kinetic meanfield approximation' (KMFA) [13-15] which neglects such fluctuations and corresponds to the replacement in (38) of each occupation operator $n(\boldsymbol{r})$ by its mean value $c(\boldsymbol{r})=\langle n(\boldsymbol{r})\rangle$ where $\langle\cdots\rangle$ means averaging over the space- and time-dependent distribution function [13-15]. Therefore, in considerations of $\mathrm{A} 1 \rightarrow \mathrm{L} 1_{0}$ transformations below, we approximate the total effective Hamiltonian $H$ in (5) by the following expression:

$H=H_{c}+H_{d 4}=H_{c}\{n(\boldsymbol{r})\}+\frac{1}{2} \sum_{\boldsymbol{r}, \boldsymbol{r}^{\prime}, \boldsymbol{R}, \boldsymbol{R}^{\prime}} c(\boldsymbol{r}) c(\boldsymbol{r}+\boldsymbol{R}) \Phi_{4}^{\boldsymbol{R}, \boldsymbol{R}^{\prime}}\left(\boldsymbol{r}-\boldsymbol{r}^{\prime}\right) c\left(\boldsymbol{r}^{\prime}\right) c\left(\boldsymbol{r}^{\prime}+\boldsymbol{R}^{\prime}\right)$

where the potential $\Phi_{4}^{R, R^{\prime}}(\boldsymbol{r})$ is given by equation (39).

Let us comment on the correspondence between the deformational interaction $H_{d 4}$ in (40) and the phenomenological expression for this elastic energy used by Chen et al [12]. Their phenomenological expression corresponds to the 'long-wavelength limit' of $H_{d 4}$ in which functions $G_{k}$ and $f_{2 k}$ in (39) are replaced by their expansions at small $k$ while the products $c(\boldsymbol{r}) c(\boldsymbol{r}+\boldsymbol{R})$ and $c\left(\boldsymbol{r}^{\prime}\right) c\left(\boldsymbol{r}^{\prime}+\boldsymbol{R}^{\prime}\right)$ in (40) are replaced by $c(\boldsymbol{r})^{2}$ and $c\left(\boldsymbol{r}^{\prime}\right)^{2}$. Therefore (saying nothing about the unrealistic models for interactions and ordering used in [12]), the phenomenological approach cannot describe features of the evolution in the short-rangeinteraction systems for which the characteristic inhomogeneities, APBs, have a microscopical width $w \lesssim a[15,16]$.

\section{Models and methods of simulation}

To simulate $\mathrm{A} 1 \rightarrow \mathrm{L}_{0}$ transformations in an alloy with the Hamiltonian (40), we use the methods described in [15] and [16], to be referred to as I and II, respectively. The evolution of the atomic distributions is described by the kinetic tetrahedron cluster field method 
(KTCFM) [15] in which mean occupations $c_{i}=c\left(\boldsymbol{r}_{i}\right)=\left\langle n\left(\boldsymbol{r}_{i}\right)\right\rangle$ averaged over the space- and time-dependent distribution function obey the kinetic equation (I.10):

$$
\mathrm{d} c_{i} / \mathrm{d} t=2 \sum_{j} M_{i j} \sinh \left[\beta\left(\lambda_{j}-\lambda_{i}\right) / 2\right] .
$$

Here $\beta=1 / T$ is the inverse temperature; $M_{i j}$ is the generalized mobility; and $\lambda_{i}=\lambda_{i}\left\{c_{j}\right\}$ is the local chemical potential equal to the derivative of the generalized free energy $F\left\{c_{i}\right\}$ defined in $[13,14]$ with respect to $c_{i}: \lambda_{i}=\partial F / \partial c_{i}$.

The expression for $M_{i j}=M_{i j}\left\{c_{k}\right\}$ employed in our simulations is given by equation (I.12) with the asymmetrical potential $u_{i}$ taken as zero for simplicity:

$$
M_{i j}=\gamma_{n n}\left[c_{i} c_{j}\left(1-c_{i}\right)\left(1-c_{j}\right)\right]^{1 / 2}
$$

where $\gamma_{n n}$ is the configurationally independent factor in the probability of an atomic exchange $\mathrm{A} i \leftrightarrow \mathrm{B} j$ between neighbouring sites $i$ and $j$ per unit time defined by equation (I.3). The simulation results below are presented in terms of the reduced time $t^{\prime}=t \gamma_{n n}$. To get an idea of the real timescale of the evolution one can use, for example, the relation between our model $\gamma_{n n}$ and the diffusivity $D(T)$ in dilute FCC alloys [21]:

$$
D(T)=a^{2} \gamma_{n n}
$$

where $a$ is the lattice constant. For example, for the Co-Pt alloys, using high-temperature estimates of diffusivity $D(T)=D_{0} \exp (-A / T)$ [22]: $D_{0} \simeq 0.6 \times 10^{-4} \mathrm{~m}^{2} \mathrm{~s}^{-1}$ and $A \simeq 2.8 \mathrm{eV}$, we obtain at $T$ near the critical temperature $T_{c} \simeq 1100 \mathrm{~K}: \gamma_{n n}(T) \simeq$ $40 \exp \left[30\left(T-T_{c}\right) / T\right] \mathrm{s}^{-1}$ (though the estimate is evidently very crude).

The local chemical potential $\lambda_{i}$ in (41) is the sum of the chemical and the deformational term, $\lambda_{i}^{c}$ and $\lambda_{i}^{d}$. The deformational contribution $\lambda_{i}^{d}=\lambda_{i}^{d}\left(\boldsymbol{r}_{i}\right)$ is the variational derivative of the second term in (40) with respect to $c_{i}=c\left(\boldsymbol{r}_{i}\right)$ :

$$
\lambda_{i}^{d}(\boldsymbol{r})=\delta H_{d 4} / \delta c(\boldsymbol{r})=2 \sum_{\boldsymbol{r}^{\prime}, \boldsymbol{R}, \boldsymbol{R}^{\prime}} c(\boldsymbol{r}+\boldsymbol{R}) \Phi_{4}^{\boldsymbol{R}, \boldsymbol{R}^{\prime}}\left(\boldsymbol{r}-\boldsymbol{r}^{\prime}\right) c\left(\boldsymbol{r}^{\prime}\right) c\left(\boldsymbol{r}^{\prime}+\boldsymbol{R}^{\prime}\right) .
$$

The chemical contribution $\lambda_{i}^{c}$ includes only chemical interactions $v_{i j}^{c}$, and in the KTCFM it has the form [15]

$\lambda_{i}=T \ln \frac{c_{i}}{1-c_{i}}+\sum_{j, n>1} T \ln \left(1-c_{j} g_{n}^{i j}\right)+\sum_{\{j k l\} \in t Y, i} T \ln \left[y_{i}^{i j k l}\left(1-c_{i}\right) / c_{i}\right]$

where the second and the third term describe the contribution of non-nearest and nearest chemical interactions, respectively. The summand in the second term corresponds to the interaction $v_{i j}^{c}=v_{n}$ between sites $i$ and $j$ which are $n$th neighbours in the lattice, and the function $g_{n}^{i j}$ is expressed in terms of the Mayer function $f_{i j}=\exp \left(-\beta v_{i j}^{c}\right)-1$ as follows:

$g_{n}^{i j}=\frac{2 f_{i j}}{R_{i j}+1+f_{i j}\left(c_{i}+c_{j}\right)} \quad R_{i j}=\left\{\left[1+\left(c_{i}+c_{j}\right) f_{i j}\right]^{2}-4 c_{i} c_{j} f_{i j}\left(f_{i j}+1\right)\right\}^{1 / 2}$.

The summand in the last sum in (45) corresponds to the contribution to $\lambda_{i}$ of the tetrahedron of neighbouring sites $i, j, k, l$, and the notation $\{j k l\} \in t Y, i$ means that the summation is performed over four 'non-overlapping' tetrahedra which contain site $i$ and have no common edges. The quantity $y_{i}^{i j k l}=y_{i}^{\alpha}\left\{c_{s}\right\}$ is determined by a system of equations:

$$
c_{i}=y_{i}^{\alpha} \partial \ln Z_{\alpha} / \partial y_{i}^{\alpha}
$$

where $Z_{\alpha}$ is the partition function for the cluster $\alpha$, which is a polynomial in $y_{s}^{\alpha}$ with $s$ equal to $i, j, k$, or $l$ :

$$
Z_{\alpha}=1+\sum_{s=i, j, k, l} y_{s}^{\alpha}+\zeta \sum_{s<s^{\prime}} y_{s}^{\alpha} y_{s^{\prime}}^{\alpha}+\zeta^{3} \sum_{s<s^{\prime}<s^{\prime \prime}} y_{s}^{\alpha} y_{s^{\prime}}^{\alpha} y_{s^{\prime \prime}}^{\alpha}+\zeta^{6} y_{i}^{\alpha} y_{j}^{\alpha} y_{k}^{\alpha} y_{l}^{\alpha}
$$


and $\zeta=\exp \left(-\beta v_{1}\right)$. Equations (47) for $y_{s}^{\alpha}$ in each cluster $\alpha$ form a system of four algebraic equations which is easily solved using Newton's method.

For the chemical interaction $v_{i j}^{c}=v_{n}$ we employ the five alloy models used in I and II:

(1) The second-neighbour interaction model with the nearest-neighbour interaction $v_{1}=$ $1000 \mathrm{~K}$ (in units of the Boltzmann constant $k_{\mathrm{B}}$ ) and $v_{2} / v_{1}=\epsilon=-0.125$.

(2) The same model with $\epsilon=-0.25$.

(3) The same model with $\epsilon=-0.5$.

(4) The fourth-neighbour interaction model with $v_{n}$ estimated by Chassagne et al [23] from their experimental data for disordered $\mathrm{Ni}-\mathrm{Al}$ alloys: $v_{1}=1680 \mathrm{~K}, v_{2}=-210 \mathrm{~K}$, $v_{3}=35 \mathrm{~K}$, and $v_{4}=-207 \mathrm{~K}$.

(5) The fourth-neighbour interaction model with $v_{1}=1000 \mathrm{~K}, v_{2} / v_{1}=-0.5, v_{3} / v_{1}=0.25$, and $v_{4} / v_{1}=-0.125$.

The effective interaction range $R_{\text {int }}$ for these models monotonically increases with the model number. Therefore, a comparison of the simulation results for these models enables one to study the influence of $R_{\text {int }}$ on the microstructural evolution. The critical temperature $T_{c}$ for the phase transition A $1 \rightarrow \mathrm{L}_{0}$ in the absence of deformational interaction $H_{d 4}$ (which seems to have little effect on $T_{c}$ in our simulations) for models $1,2,3,4$, and 5 is 614,840 , 1290,1950 , and $2280 \mathrm{~K}$, respectively [24].

For the Kanzaki force $f_{2 k}^{R}$ entering the expression (39) for the potential $\Phi_{4}^{R, R^{\prime}}(\boldsymbol{r})$ in (44) we use equations (34) and (35). The interaction parameters $\varphi_{a}$ and $\varphi_{b}$ in these equations can be expressed via spontaneous deformations $\bar{u}$ and $\bar{\varepsilon}$ using equations (29) and (32). For simplicity we assume the volume striction to be small (as it is for the Co-Pt alloys mentioned above): $\bar{u} \simeq 0$, while the tetragonal distortion will be characterized by its maximum value $\varepsilon_{m}$ in a stoichiometric alloy, i.e. by the value $\bar{\varepsilon}$ in (29) at $\eta_{1}=0.5$. Therefore, interactions $\varphi_{a}$ and $\varphi_{b}$ in our simulations are determined by the relations

$$
\varphi_{a}=-a\left(c_{11}-c_{12}\right) \varepsilon_{m} / 3 \quad \varphi_{b}=a\left(c_{11}-c_{12}\right) \varepsilon_{m} / 12 .
$$

For the lattice constant $a$ in (49) we take a typical value $a \simeq 4 \AA$, and for the elastic constant $\left(c_{11}-c_{12}\right)$, the value 0.97 Mbar corresponding to FCC platinum [27].

For the force constant matrix $\bar{A}_{k}$ (which determines the matrix $G_{k}=\left(\bar{A}_{k}\right)^{-1}$ in equation (39)) we use the model described in [15,28]. It corresponds to a Born-von Karman model with the first- and second-neighbour force constants only, and the second-neighbour constants are supposed to correspond to a spherically symmetrical interaction. This model includes three independent force constants which are expressed in terms of elastic constants $c_{i k}$, and these constants were chosen equal to those of FCC platinum [27]: $c_{11}=3.47 \mathrm{Mbar}$, $c_{12}=2.5$ Mbar, and $c_{44}=0.77$ Mbar.

As was discussed in I and II, the transient partially ordered alloy states can be described using either mean occupations $c_{i}=c\left(\boldsymbol{r}_{i}\right)$ or local order parameters $\eta_{\alpha i}^{2}$ and local concentrations $\bar{c}_{i}$ defined by equations (I.24) and (I.25). The simulation results below are usually presented as the distributions of quantities $\eta_{i}^{2}=\eta_{1 i}^{2}+\eta_{2 i}^{2}+\eta_{3 i}^{2}$, to be called the ' $\eta^{2}$-representation', and these distributions are similar to those observed in experimental transmission electron microscopy (TEM) images [15].

Our simulations were performed on FCC simulation boxes of sizes $V_{b}=L^{2} \times H$ (where $L$ and $H$ are given in units of the lattice constant $a$ ) with periodic boundary conditions. We used both quasi-2D simulations with $H=1$ and 3D simulation with $H \sim L$. For the given coordinate $z=n a$ (with $n=0$ for 2D simulation) each of the figures below shows all FCC lattice sites lying in two adjacent planes, $z=n a$ and $z=(n+1 / 2) a$. The point $(x, y)$ with $(x / a, y / a)$ equal to $(l, m),(l+1 / 2, m),(l+1 / 2, m+1 / 2)$, or $(l, m+1 / 2)$ in the figures 
corresponds to the lattice site with $(x / a, y / a, z / a)$ equal to $(l, m, n),(l+1 / 2, m, n+1 / 2)$, $(l+1 / 2, m+1 / 2, n)$, or $(l, m+1 / 2, n+1 / 2)$, respectively. Therefore, at $V_{b}=L^{2} \times H$ the figure shows $4 L^{2}$ lattice sites.

The simulation methods were the same as in I and II. In simulations of A $1 \rightarrow \mathrm{L} 1_{0}$ transformation the initial as-quenched distribution $c_{i}(0)$ was characterized by its mean value $c$ and small random fluctuations $\delta c_{i}$; usually we used $\delta c_{i}= \pm 0.01$. The distributions of initial fluctuations $\delta c_{i}$ for the given simulation box volume $V_{b}$ were identical for all models and the same as that used in II. The sensitivity of the simulation results to variations of these initial fluctuations $\delta c_{i}$ was discussed in II and was found to be insignificant for the features of evolution discussed below.

\section{Kinetics of $\mathrm{A} 1 \rightarrow \mathrm{L1}_{0}$ transformations in systems with an extended or intermediate interaction range}

As discussed in I, II, and below, features of microstructural evolution under $\mathrm{A} 1 \rightarrow \mathrm{L} 1_{0}$ and $\mathrm{A} 1 \rightarrow \mathrm{L} 1_{2}$ transitions sharply depend on the effective interaction range $R_{\text {int }}$ in an alloy. In this section we discuss $\mathrm{A} 1 \rightarrow \mathrm{L} 1_{0}$ transitions for the systems with an extended or intermediate interaction range, such as our models 5 and 4, while the short-range-interaction systems are considered in the next section.

Some results of our simulations are presented in figures 1-8. The symbol A or $\bar{A}$ in these figures corresponds to an $\mathrm{L1}_{0}$-ordered domain with the tetragonal axis $c$ along (100) and the positive or negative value, respectively, of the order parameter $\eta_{1}$; the symbol B or $\overline{\mathrm{B}}$ corresponds to that for the $c$-axis along (010) and the order parameter $\eta_{2}$; and the symbol $\mathrm{C}$ or $\overline{\mathrm{C}}$ corresponds to that for the $c$-axis along $(001)$ and the order parameter $\eta_{3}$. Figure 8 shown in the $c$-representation illustrates the occupation of lattice sites for each domain type. The APB separating two APDs with the same tetragonal axis (i.e. APDs A and $\overline{\mathrm{A}}, \mathrm{B}$ and $\overline{\mathrm{B}}$ or $\mathrm{C}$ and $\overline{\mathrm{C}}$ ) will be for brevity called the 'shift-APB', and the APB separating the APDs with perpendicular tetragonal axes will be called the 'flip-APB'.

\subsection{Stages of $A_{1} \rightarrow L_{10}$ transformation}

Before discussing figures 1-8 we recall the general ideas about the formation of twinned structures [1-11]. To avoid discussing the problems of nucleation, in this work we consider transformation temperatures $T$ lower than the ordering spinodal temperature $T_{s}$. Then the evolution under $\mathrm{A} 1 \rightarrow \mathrm{L}_{0}$ transition includes the following stages [2-6]:

(i) The initial stage of the formation of the finest $\mathrm{L}_{1}$-ordered domains when their tetragonal distortion still has little effect on the evolution and all six types of APD are present in microstructures in the same proportion. It corresponds to the so-called 'mottled' contrast in TEM images $[5,6]$.

(ii) The next, intermediate stage which corresponds to the so-called 'tweed' contrast in TEM images. The tetragonal deformation of the $\mathrm{L} 1_{0}$-ordered APDs here leads to the predominance of the (110)-type orientations of flip-APBs, but all six types of APD (i.e. APDs with all three orientations of the tetragonal axis $c$ ) are still present in microstructures in comparable proportions [2-4].

(iii) The final, polytwinned stage when the tetragonal distortion of the $\mathrm{L} 1_{0}$-ordered APDs becomes the main factor in the evolution and leads to the formation of (110)-type oriented twin bands. Each band includes only two types of APD with the same $c$-axis, and these axes in the adjacent bands are 'twin' related, i.e. have alternate (100) and (010) orientations for the given set of the (110)-oriented bands [2-4]. 


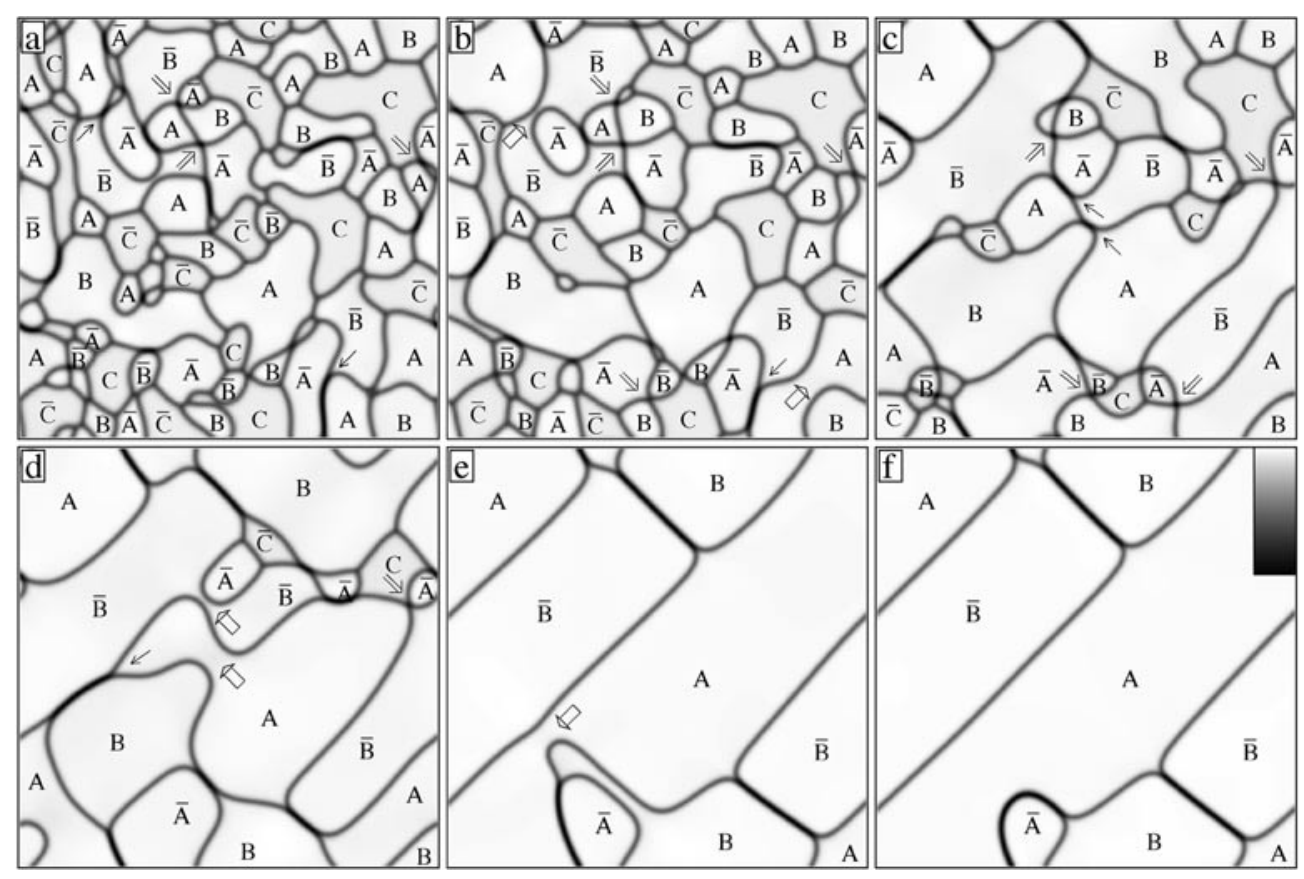

Figure 1. Temporal evolution of extended-interaction-range model 5 under the phase transformation $\mathrm{A} 1 \rightarrow \mathrm{L}_{0}$ shown in the $\eta^{2}$-representation for the simulation box size $V_{b}=128^{2} \times 1$ for the maximum tetragonal distortion parameter $\left|\varepsilon_{m}\right|=0.1, c=0.5$, the reduced temperature $T^{\prime}=T / T_{c}=0.7$, and the following values of the reduced time $t^{\prime}=t \gamma_{n n}$ : (a) 10 ; (b) 20 ; (c) 50 ; (d) 100 ; (e) 250 ; and (f) 280 . The grey level varies linearly with $\eta_{i}^{2}=\eta_{1 i}^{2}+\eta_{2 i}^{2}+\eta_{3 i}^{2}$ between its minimum and maximum values, $\eta_{\min }^{2}<10^{-4}$ and $\eta_{\max }^{2} \simeq 0.20$, from completely dark to completely bright as shown in the inset. The symbol $\mathrm{A}, \overline{\mathrm{A}}, \mathrm{B}, \overline{\mathrm{B}}, \mathrm{C}$ or $\overline{\mathrm{C}}$ indicates the type of the ordered domain as described in the text. The thick, the thin, and the single arrow indicate the fusion-of-domain process, the quadruple junction of APDs, and the splitting APB process, respectively, as discussed in the text.

The thermodynamic driving force for the (110)-type orientation of flip-APBs is the gain in the elastic energy of adjacent APDs: at other orientations this energy increases with the growth of an APD proportionally to its volume [8-11]. For an APD with the characteristic size $l$ and the surface $S_{d}$, this elastic energy $E_{\mathrm{el}}^{v} \sim c_{-} \bar{\varepsilon}^{2} S_{d} l$ begins to affect the microstructural evolution when it becomes comparable with the surface energy $E_{s} \sim \sigma S_{d}$ where $\sigma$ is the APB surface tension. The 'tweed' stage (ii) corresponds to the relation $E_{\mathrm{el}}^{v} \sim E_{s}$ or to the characteristic APD size

$$
l_{0} \sim \sigma / c_{-} \bar{\varepsilon}^{2}
$$

and so this size sharply increases with decreasing distortion $\bar{\varepsilon}$.

Figures 1-7 illustrate quasi-2D simulations for which microstructures include only edgeon APBs normal to the (001) plane. The elimination of the volume-dependent elastic energy mentioned above is here possible only for the (100)- and (010)-oriented APDs separated by the (110)- or (1 $\overline{1} 0)$-oriented APBs, while in the (001)-oriented APDs $\mathrm{C}$ and $\overline{\mathrm{C}}$ this elastic energy is always present. Therefore, the tweed stage (ii) in these simulations corresponds to both the predominance of (110)- or (110)-oriented APBs separating domains A or $\bar{A}$ from $\mathrm{B}$ or $\overline{\mathrm{B}}$ and the decrease of the portion of domains $\mathrm{C}$ and $\overline{\mathrm{C}}$ in the microstructures. In the $3 \mathrm{D}$ case each of three possible types of a polytwin, those without (001)-, (100)-, or (010)- 


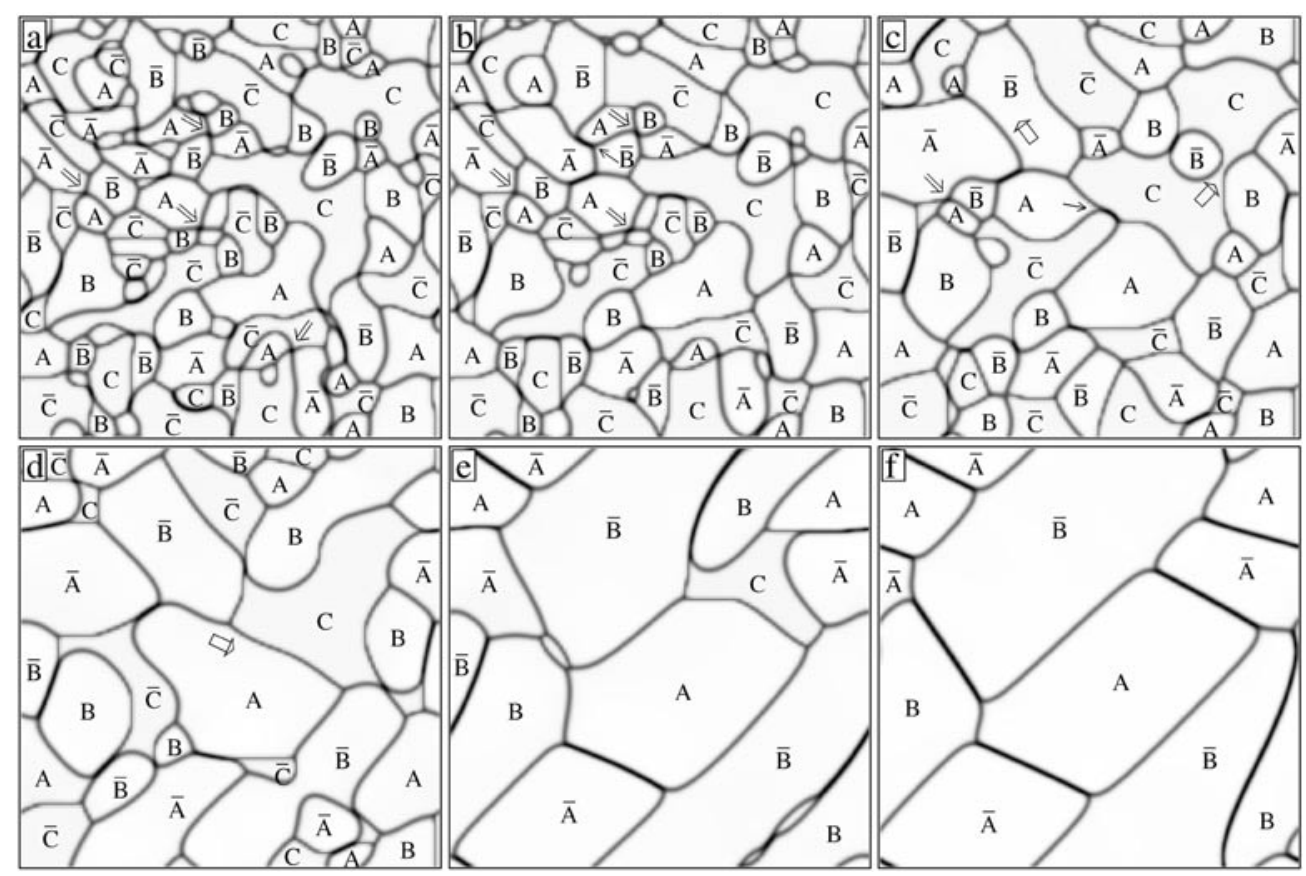

Figure 2. As figure 1, but for intermediate-interaction-range model 4 at $T^{\prime}=0.67, \eta_{\max }^{2} \simeq 0.23$, and the following values of $t^{\prime}$ : (a) 10; (b) 20; (c) 50; (d) 100; (e) 250; and (f) 500 .

oriented APDs, can be formed in the given part of an alloy stochastically due to the local fluctuations of composition [1-7]. This is illustrated, in particular, by the 3D simulation shown in figure 8 , while quasi-2D simulations reproduce the formation of only one of the polytwin types mentioned above.

The distortion parameter $\left|\varepsilon_{m}\right|=0.1$ for the simulations shown in figures $1-3$ was chosen such that the APD size $l_{0}$ in equation (50) characteristic for manifestations of elastic effects has the scale typical for real CoPt-type alloys. In particular, if we make the conventional assumption that the APB energy $\sigma$ is proportional to the transition temperature $T_{c}: \sigma \sim T_{c} f\left(T^{\prime}\right)$ where $f$ is some function of the reduced temperature $T^{\prime}=T / T_{c}$, then using the relation $\varepsilon_{m}=\bar{\varepsilon} / 4 \eta_{1}^{2}$ and the parameters $\bar{\varepsilon}, \eta_{1}$, and $T_{c}$ for CoPt and for our models mentioned above, we find that the right-hand side of equation (50) for models 5 and 4 at $\left|\varepsilon_{m}\right|=0.1$ is close to that for the CoPt alloy at similar $T^{\prime}$-values within about $10 \%$. Therefore, the microstructures at both the initial stage (i) and the tweed stage (ii) can be reproduced by figures $1-3$ with no significant distortion of scales. With further growth of an APD its size $l$ becomes comparable with the simulation box size $L$, and the periodic boundary conditions begin to significantly affect the evolution. Therefore, the later stages of transformation can be more adequately simulated if we reduce the characteristic size $l_{0}$ in equation (50) using the larger values of the parameter $\varepsilon_{m}$, such as $\left|\varepsilon_{m}\right|=0.15-0.2$ used in the simulations shown in figures 4-8.

\subsection{Features of evolution at the initial and tweed stages}

Let us first discuss figures 1-3 corresponding to a 'realistic' distortion parameter $\left|\varepsilon_{m}\right|=0.1$. The initial stage (i) in these figures corresponds to frames 1(a), (b), 2(a), (b), and 3(a), (b); the tweed stage (ii) corresponds to frames 1(c), (d), 2(c)-(e), and 3(c); and the twin stage (iii) corresponds to frames 1(e), (f), 2(f), and 3(d). 


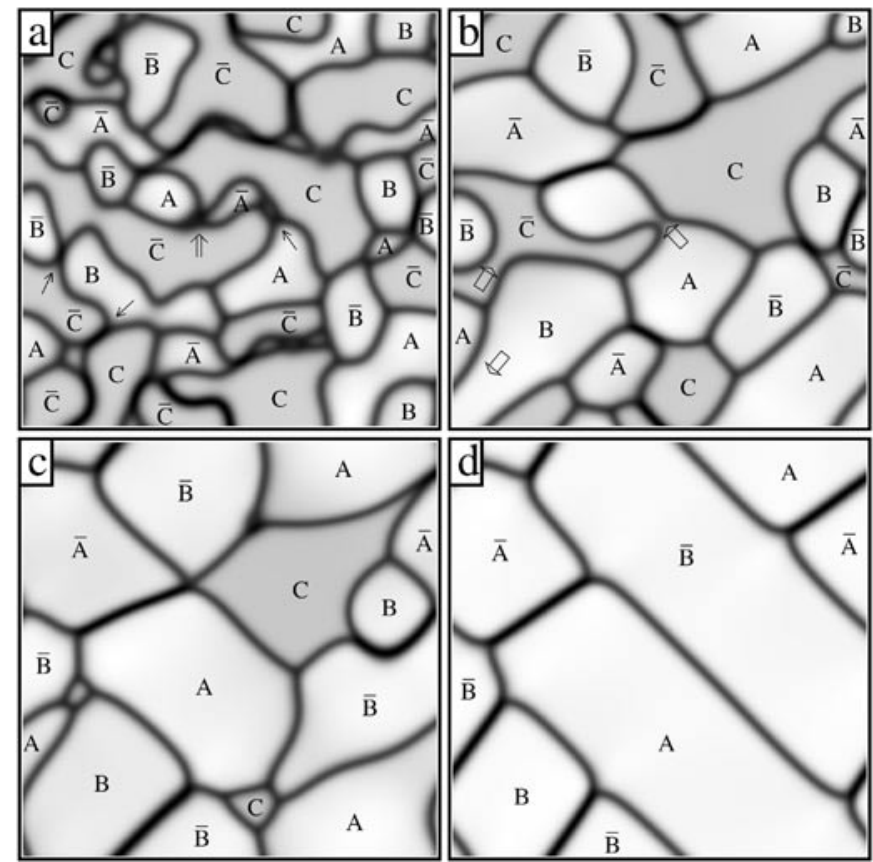

Figure 3. As figure 2, but at $T^{\prime}=0.92, \eta_{\max }^{2} \simeq 0.14$, and the following values of $t^{\prime}$ : (a) 10 ; (b) 50; (c) 100; and (d) 200 .

The detailed consideration of the initial stage for models 4 and 5 neglecting the deformational effects [16] revealed the following features of evolution:

(a) The presence of abundant processes of fusion of in-phase domains which are among the main mechanisms of domain growth at this stage.

(b) The presence of peculiar long-living configurations, the quadruple junctions of APDs (4junctions) of the type $A_{1} A_{2} \overline{A_{1}} A_{3}$ where $A_{2}$ and $A_{3}$ can correspond to any two of four types of APD different from $\mathrm{A}_{1}$ and $\overline{\mathrm{A}_{1}}$.

(c) The presence of many processes of 'splitting' of a shift-APB into two flip-APBs which lead to either the fusion of in-phase domains mentioned in point (a) ( $\mathrm{s} \rightarrow \mathrm{f}$ process) or the formation of a 4 -junction mentioned in point $(\mathrm{b})(\mathrm{s} \rightarrow 4 j$ process).

Figures 1-3 show that all these microstructural features are also present when the deformational effects are taken into account, and not only at the initial stage (i) but also at the tweed stage (ii). In particular, the beginning and the end of an $\mathrm{s} \rightarrow \mathrm{f}$ process (marked by the single and the thick arrow, respectively) can be followed in frames 1(a)-(d); 1(d) and (e); 2(b) and (c); 2(c) and (d); and 3(a) and (b). The fusion with the disappearance of an intermediate APD which initially separates two in-phase domains to be fused [16] can be followed in the lower right part of frames 1(a) and (b) and in the upper right part of frames 2(b) and (c) (which is marked by a thick arrow in frames 1(b) and 2(c), respectively). A number of longliving 4-junctions marked by thin arrows are seen in frames 1(a)-(d), 2(a)-(c), and 3(a). An $\mathrm{s} \rightarrow 4 j$ process can be followed in the lower right part of frames 1 (a)-(c). The processes and configurations (a), (b), and (c) can also be seen in figures 4-7 discussed below.

Frames 2(a)-(e) also display some (100)-oriented and thin conservative APBs. As discussed in [16] and below, such APBs are most typical of the short-range-interaction systems 


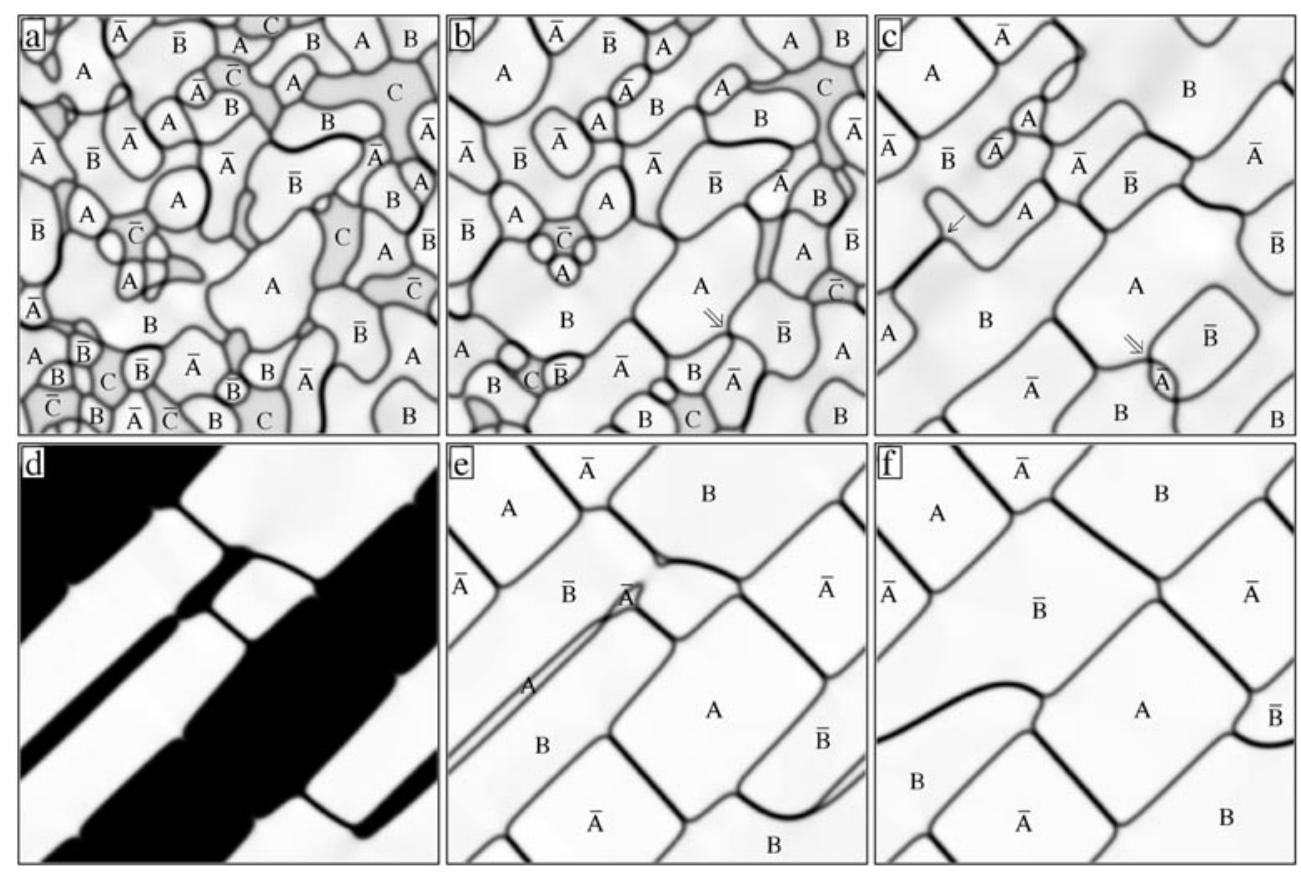

Figure 4. As figure 1, but at $\left|\varepsilon_{m}\right|=0.15, \eta_{\max }^{2} \simeq 0.21$, and the following values of $t^{\prime}$ : (a) 10 ; (b) 20; (c) 50 ; (d) 150 ; (e) 172; and (f) 350. Frame 2(d) is shown in the $\eta_{2}^{2}$-representation: the grey level varies linearly with $\eta_{2 i}^{2}$ between its minimum and maximum values, $\eta_{2 \min }^{2}<10^{-7}$ and $\eta_{2 \max }^{2} \simeq 0.21$, from completely dark to completely bright.

where they have a low surface energy (being zero for the stoichiometric nearest-neighbour interaction model) unlike other, non-conservative APBs. For increase of the interaction range, as well as temperature, or deviation from the stoichiometric composition, the anisotropy in the APB surface energy sharply decreases [16]. Therefore, in figure 2 (and figure 5 below) corresponding to intermediate-range-interaction model 4 the conservative APBs are few but observable, while for extended-range-interaction model 5 in figure 1 , as well as at elevated $T$ or for significant 'non-stoichiometry' $\delta c=(0.5-c)$ in figures 3 or 6 for model 4 , such APBs are absent entirely.

Comparison of figures 2 and 3 reveals the sharp dependence of microstructural evolution on the transformation temperature $T$. Upon elevating this temperature to values near the critical temperature $T_{c}:\left(T_{c}-T\right) \lesssim 0.1 T_{c}$, both flip- and shift-APBs notably thicken, the anisotropy in their surface energy falls off, while the characteristic size of the initial APDs (formed after a rapid quench $\mathrm{A} 1 \rightarrow \mathrm{L} 1_{0}$ ) increases. The latter change is related to an increase at $T \rightarrow T_{c}$ of the characteristic wavelength for the ordering instability which is due to the narrowing of the interval of effective wavenumbers $\boldsymbol{q}=\boldsymbol{k}-\boldsymbol{k}_{s}$ near the superstructure vector $\boldsymbol{k}_{s}$ for which the ordering concentration waves are unstable at $T<T_{c}$.

Frames 1(c), (d), 2(c)-(e), and 3(c) show the evolution at the tweed stage. They illustrate, in particular, the kinetics of the (110)-type alignment of APBs between APDs A or $\bar{A}$ and B or $\overline{\mathrm{B}}$ at this stage, as well as a 'dying out' of (100)-oriented APDs $\mathrm{C}$ and $\overline{\mathrm{C}}$. These frames also show that in the simulation with a realistic distortion parameter $\left|\varepsilon_{m}\right|=0.1$ (fitted to the structural data for CoPt), the APD size $l_{0}(50)$ characteristic of the tweed stage is about (20-40) $a$. This agrees with the order of magnitude of this size observed in the CoPt-type alloys FePt and FePd [2-4]. 


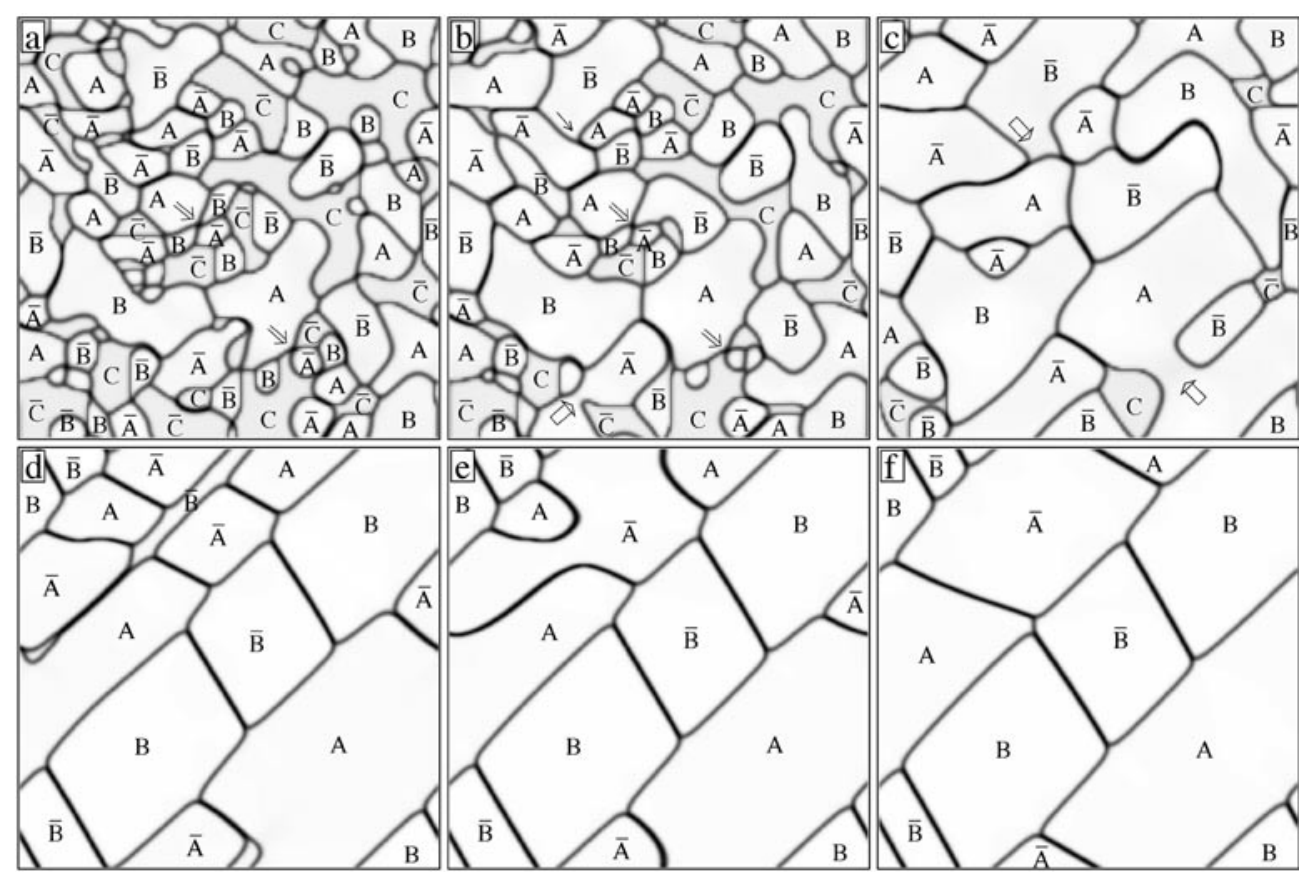

Figure 5. As figure 2, but at $\left|\varepsilon_{m}\right|=0.15, \eta_{\max }^{2} \simeq 0.24$, and the following values of $t^{\prime}$ : (a) 10 ; (b) 20; (c) 50; (d) 170; (e) 200; and (f) 700 .

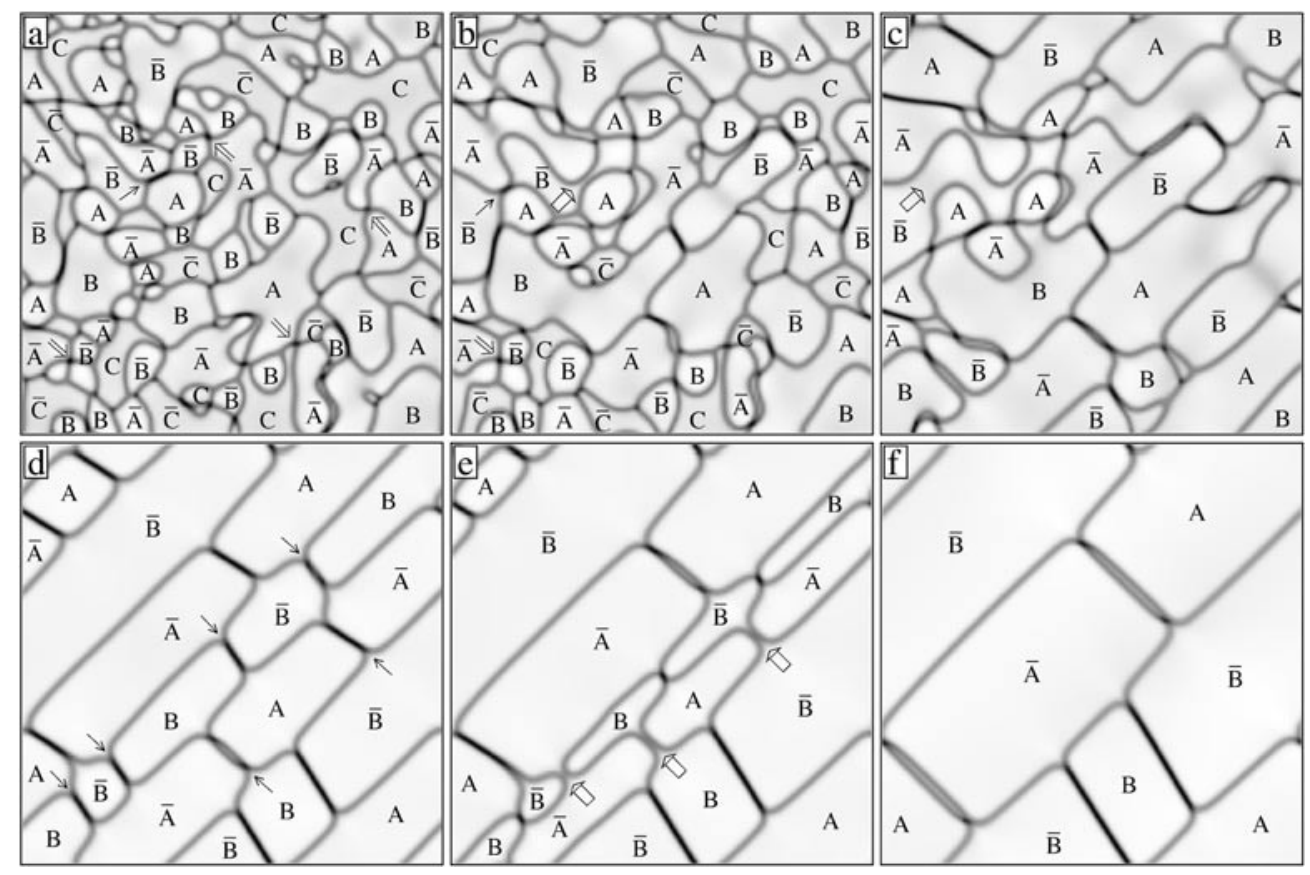

Figure 6. As figure 5, but at $c=0.44, \eta_{\max }^{2} \simeq 0.20$, and the following values of $t^{\prime}$ : (a) 10 ; (b) 20 ; (c) 50; (d) 400; (e) 750; and (f) 1100 . 


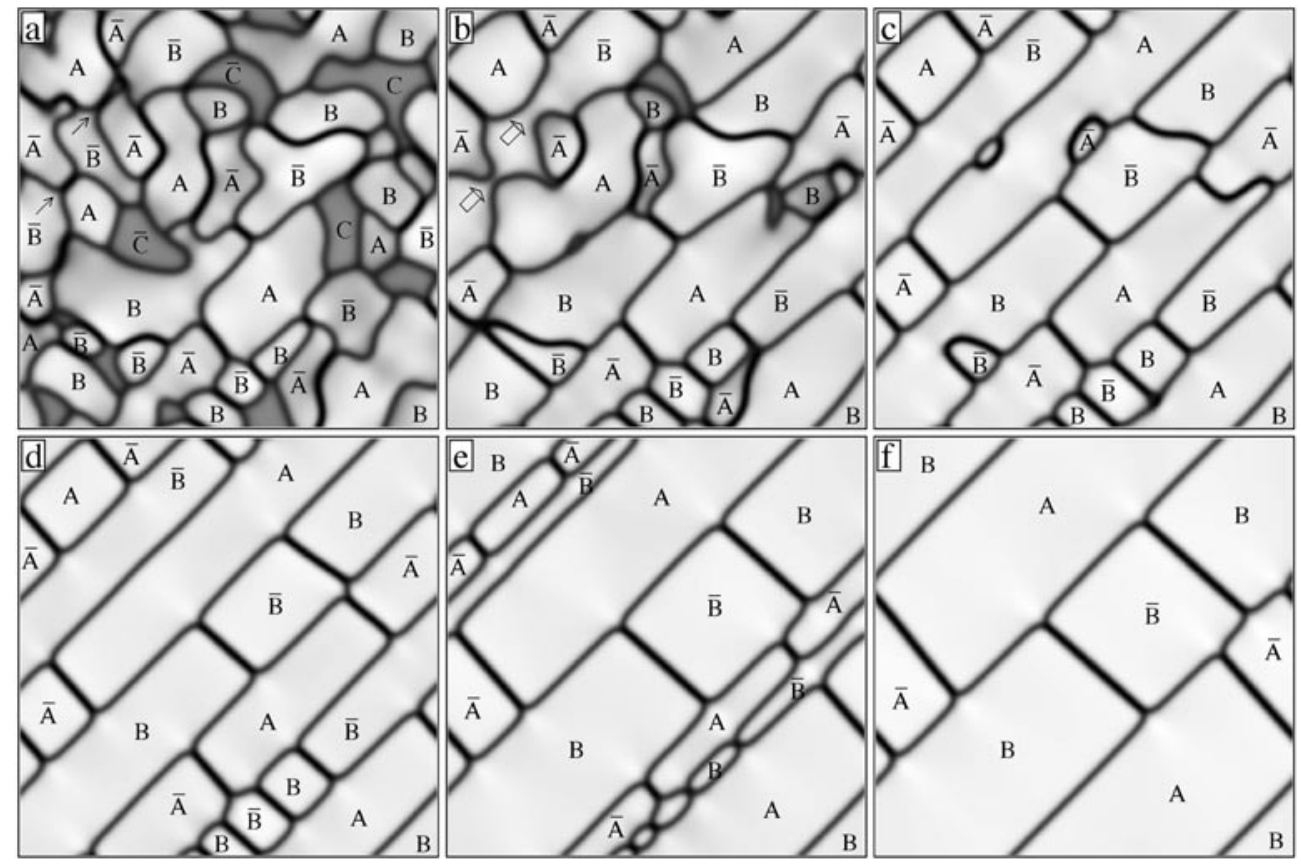

Figure 7. As figure 1, but at $\left|\varepsilon_{m}\right|=0.2, T^{\prime}=0.88, \eta_{\max }^{2} \simeq 0.17$, and the following values of $t^{\prime}$ : (a) 10; (b) 20; (c) 30; (d) 50; (e) 300; and (f) 400 .

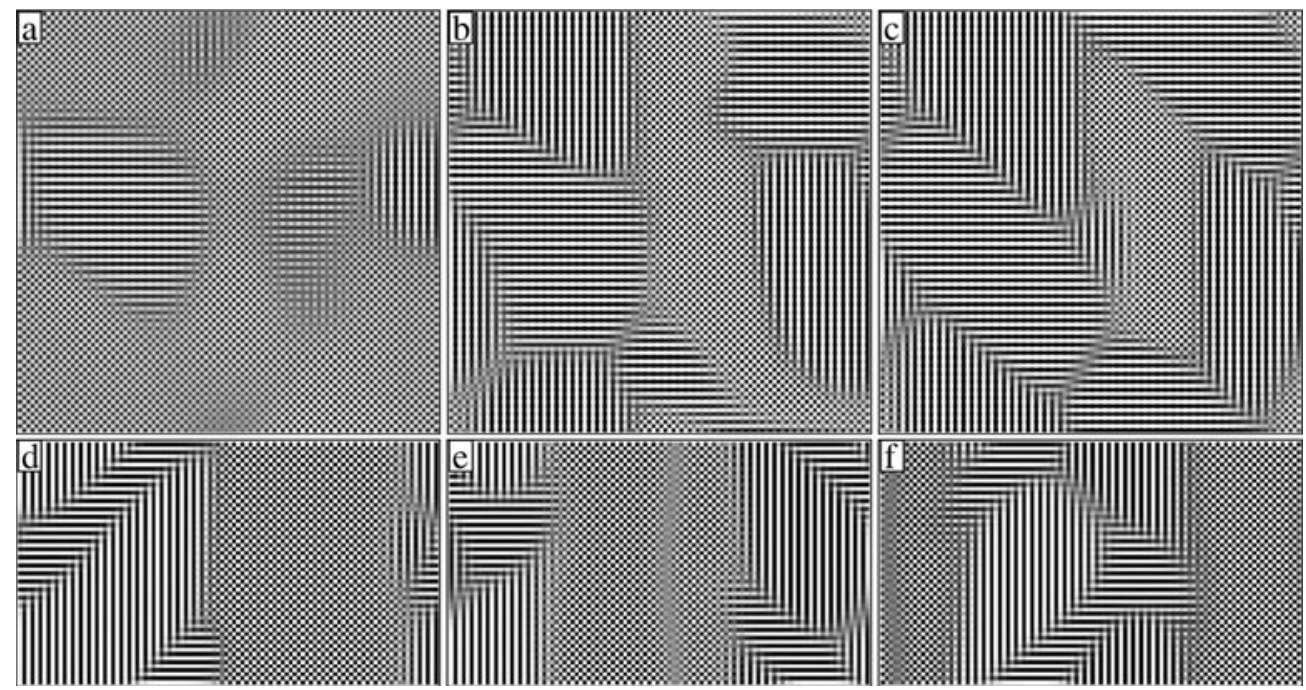

Figure 8. As figure 3, but for 3D simulation with $V_{b}=52^{2} \times 30$ at $\left|\varepsilon_{m}\right|=0.2$ and shown in the ' $c$-representation': the grey level varies linearly with $c_{i}$ between its minimum and maximum values, $c_{\min } \simeq 0.04$ and $c_{\max } \simeq 0.96$, from completely dark to completely bright. The upper three frames correspond to the plane $z=10 a$ and the following values of $t^{\prime}$ : (a) 10; (b) 20; and (c) 325 . The lower three frames correspond to $t^{\prime}=325$ and the following planes: (d) $y=0$; (e) $y=10 a$; and (f) $y=36 a$. 


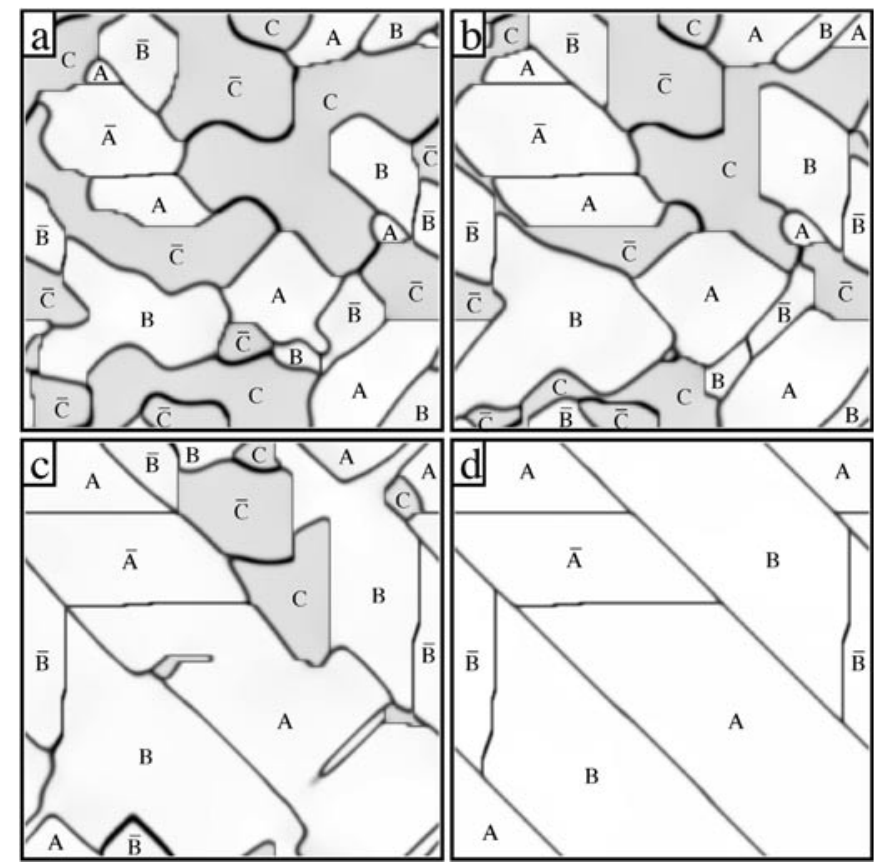

Figure 9. As figure 1, but for the short-range-interaction model 1 at $\left|\varepsilon_{m}\right|=0.15, c=0.5, T^{\prime}=0.9$, $\eta_{\max }^{2} \simeq 0.24$, and the following values of $t^{\prime}$ : (a) 30; (b) 40; (c) 60; and (d) 120 .

\subsection{Microstructural evolution at the twin stage}

As mentioned, the final, twin stage of the transformation can be more adequately simulated with the larger values of the parameter $\left|\varepsilon_{m}\right|$ which are employed in the simulations shown in figures 4-8. Before discussing these figures we note some typical configurations observed in experimental studies of transient twinned microstructures [2-4] seen, for example, in figures 5, 6,9 , and 2 in [2-4] and [12], respectively:

(1) semi-loop-like shift-APBs adjacent to the twin band boundaries;

(2) 'S-shaped' shift-APBs stretching across the twin band; and

(3) short and narrow twin bands (for brevity to be called 'microtwins') which lie within the larger twin bands and usually have one or two shift-APBs near their edges.

Comparing our results with experiments one should consider that due to the limited size of the simulation box the twin bandwidth $d$ in our simulations has the same order of magnitude as the APD size $l_{0}(50)$ characteristic of the tweed stage, while in experiments $d$ usually far exceeds $l_{0}[2-4]$. Therefore, the distribution of shift-APBs within twin bands in our simulation is usually much closer to equilibrium than in experiments. In spite of this difference, the simulations reproduce all of the characteristic transient configurations (1)-(3) and elucidate the mechanisms of their formation. In particular, both the semi-loop and the S-shaped shift-APBs are formed from regular-shaped approximately quadrangular APDs (characteristic of the beginning of the twin stage) due to the disappearance of adjacent APDs which are 'wrongly oriented' with respect to the given twin band. The formation of semi-loop configurations is illustrated by frames 1(d)-(f), 5(d), (e), and 7(b), (c); while the formation of S-shaped APBs can be seen in frames 2(d)-(f), 4(d)-(f), 5(d), (e), and 7(b), (c). The formation and evolution of microtwins is 
illustrated by frames 4(c), (d) and 5(c), (d). These frames show that the microtwin is actually a small and narrow APD for which deformational effects are strong enough to align its flipAPBs along the (110)-type directions. However, the standard mechanism of coarsening via the growth of larger APDs at the expense of smaller ones leads to the shrinking and eventually to the disappearance of a microtwin which is usually accompanied by the formation of S-shaped and/or semi-loop shift-APBs. The latter is illustrated by frames 4(d)-(f) and 5(d), (e). Let us also note that the microtwin configuration shown in frame $4(\mathrm{~d})$ is strikingly similar to that seen in the central part of the experimental figure 2 in [12].

Let us now discuss the final, 'nearly equilibrium' microstructures shown in the last frames of figures 1-8. A characteristic feature of these microstructures is a peculiar alignment of shift-APBs: within a (100)-oriented twin band in a (110)-type polytwin the APBs tend to align normally to some direction $n=(\cos \alpha, \sin \alpha, 0)$ characterized by a 'tilting' angle $\alpha$ between the band orientation and the APB plane. Figures $1-8$ show that this tilting angle is not very sensitive to the variations of temperature or concentration but it sharply depends on the interaction type, particularly on the interaction range. For extended-range-interaction model 5 this angle is close to $\pi / 4$ (slightly exceeding this value), while for intermediate-range-interaction model 4 it is notably less than $\pi / 4$. A similar alignment of APBs for the short-range-interaction systems is illustrated by figures 9-11 where the tilting angle is close to zero.

A phenomenological theory of this interaction-dependent tilting of APBs within nearly equilibrium twin bands has been suggested in [11]. The tilting is explained by the competition between the anisotropy of the APB surface tension $\sigma$ and a tendency to minimize the total APB area within the given twin band which corresponds to $\alpha=\pi / 4$. For the alloy systems with both the intermediate and the short interaction range the surface tension $\sigma(\alpha)$ is minimal at $\alpha=$ $0[11,16]$. Thus for such alloy systems the tilting angle is less than $\pi / 4$, and it decreases with the decrease of the interaction range. For the extended-range-interaction systems the anisotropy of the APB surface tension is weak $[15,16]$, and so the tilting angle is close to $\pi / 4$. Therefore, the comparison of experimental tilting angles with theoretical calculations [11] can provide both qualitative and quantitative information on the effective chemical interactions in an alloy.

The alignment of shift-APBs discussed above seems to be clearly seen in the experimental microstructure for CoPt shown in figure 5 of [1] where the tilting angle is notably less than $\pi / 4$. It can indicate that the effective interactions in CoPt have an intermediate interaction range. This agrees with the usual estimates of these interactions for transition metal alloys; see e.g. [19, 23, 29].

Comparison of figures 4 and 7 illustrates the influence of temperature $T$ on the evolution. Elevating $T$, we again observe a thickening of APBs, as well as a coarsening of initial APDs. Frames 7(d)-(f) also illustrate a process of 'transverse coarsening' of twin bands via a shrinkage and disappearance of some microtwinned bands. Such transverse coarsening appears to be seen in a number of experimental microstructures, for example, in figure 6 in [3] or figure 2 in [12]. Frames 7(d)-(f) show that the thermodynamic driving force for such transverse coarsening is mainly the gain in the surface energy of shift-APBs due the decrease of their total area in this process.

Figures 5 and 6 illustrate the concentration dependence of the evolution. The nonstoichiometry $\delta c=(0.5-c)$ affects the evolution similarly to temperature $T$ : for increase of both $\delta c$ and $T$ all APBs thicken, while shift-APBs become less stable with respect to flipAPBs [16]. The latter leads to an enhancement of processes of splitting of shift-APBs as well as of the transverse coarsening mentioned above; this is illustrated by frames 6(b)-(f).

Some results of a 3D simulation with $V_{b}=52^{2} \times 30$ are presented in figure 8 . In this figure we employ the $c$-representation (described in the caption) in which the regions containing the vertical or horizontal lines (that is, the vertical or horizontal crystal planes filled by A atoms) 
correspond to the APDs with the (100)- or (010)-oriented tetragonal axis, respectively, while the checkered regions correspond to the APDs with the tetragonal axis normal to the plane of the figure. This simulation aimed mainly to complement $2 \mathrm{D}$ simulations with an illustration of geometrical features of 3D microstructures. Figure 8 illustrates, in particular, a stochastic formation of different polytwin sets with three possible types of orientation mentioned above. The limited size of the simulation box prevents us from carrying out a detailed consideration of the evolution with this 3D simulation. Therefore, below, we discuss only the problem of a $3 \mathrm{D}$ orientation of tilted shift-APBs in final, 'nearly equilibrium' microstructures.

Let us consider a (100)-oriented twin band in the form of a plate of height $h$, length $l$, and width $d$ in the direction (001), (110), and (110), respectively, with $d \lesssim h \ll l$ (which is a typical form of twin bands observed in TEM experiments [1-7]). The equilibrium orientation of a plane shift-APB in this band corresponds to the minimum of its energy $E_{S}=\sigma S$ where $S$ is the APB area and $\sigma$ is the surface tension determined mainly by the angle $\alpha$ between the APB orientation $\boldsymbol{n}=(\sin \alpha, \cos \alpha \cos \varphi, \cos \alpha \sin \varphi)$ and the band orientation $\boldsymbol{n}_{0}=(100)$ [11]. For the 'needle-shaped' twin band under consideration, the upper and the lower boundaries of a shift-APB usually lie at the top and the bottom edge of this band, respectively. Minimization of energy $E_{s}$ in this case yields $\varphi=0$, i.e. the APB is normal to the (001) plane, and its orientation $\boldsymbol{n}=(\sin \alpha, \cos \alpha, 0)$ is determined by the interaction-dependent tilting angle $\alpha$ defined in [11]. This conclusion seems to be supported by the present 3D simulation: the lower and the upper tilted shift-APBs within the (010)-oriented twin band below the main diagonal of frame 8(c) correspond to the grey lines stretching across the checkered region in frames $8(\mathrm{e})$ and (f), respectively, and both of these lines are approximately normal to the (001) plane.

\section{Kinetic features of $\mathrm{A} 1 \rightarrow \mathrm{L1}_{0}$ transformations in the short-range-interaction systems}

As mentioned, transient microstructures under $\mathrm{L}_{0}$ ordering for the short-range-interaction systems include many conservative APBs. Such APBs are virtually immobile, and so the evolution is realized via motion of other, non-conservative APBs which results in a number of peculiar kinetic features $[15,16]$. The initial stage of the $\mathrm{A} 1 \rightarrow \mathrm{L} 1_{0}$ transformation for the short-range-interaction systems was discussed in detail in [16]. In this section we consider the tweed and twin stages of such transformations and note the differences from the case of systems with the larger interaction range.

Some results of our simulations for the short-range-interaction systems are presented in figures 9-11. In these simulations we used sufficiently high temperatures $T^{\prime} \gtrsim 0.9-$ 0.8 to accelerate evolution to final, 'nearly equilibrium' configurations, as the presence of conservative APBs slows down (or even 'freezes') this evolution, particularly at low $T^{\prime}$.

Figure 9 illustrates the evolution for model 1; as discussed in [15], this model seems to correspond to the $\mathrm{Cu}-\mathrm{Au}$-type alloys. A distinctive feature of microstructures shown in figure 9 is a predominance of the above-mentioned conservative APBs with the (100)-type orientation. Frames 9(a)-(c) show both the conservative shift-APBs (c-shift APBs) and the conservative flip-APBs (c-flip APBs) also illustrating their orientational properties [16]. For quasi-2D microstructures with edge-on APBs shown in figure 9, c-shift APBs separating APDs $\mathrm{A}$ and $\overline{\mathrm{A}}$ (c-APBs $\mathrm{A}-\overline{\mathrm{A}})$ are horizontal; $\mathrm{c}-\mathrm{APBs} \mathrm{B}-\overline{\mathrm{B}}$ are vertical; and c-APBs $\mathrm{C}-\overline{\mathrm{C}}$ can be both horizontal and vertical; c-flip APBs $(\mathrm{A}$ or $\overline{\mathrm{A}})-(\mathrm{C}$ or $\overline{\mathrm{C}})$ (which separate APDs $\mathrm{A}$ or $\overline{\mathrm{A}}$ from $\mathrm{C}$ or $\overline{\mathrm{C}})$ are horizontal; c-APBs $(\mathrm{B}$ or $\overline{\mathrm{B}})-(\mathrm{C}$ or $\overline{\mathrm{C}})$ are vertical; and c-APBs $(\mathrm{A}$ or $\overline{\mathrm{A}})-(\mathrm{B}$ or $\overline{\mathrm{B}})$ should lie in the plane of the figure and thus they are not seen in figure 9 . Figure 9 also shows that the conservative APBs are notably thinner than non-conservative ones, particularly for c-flip APBs. 


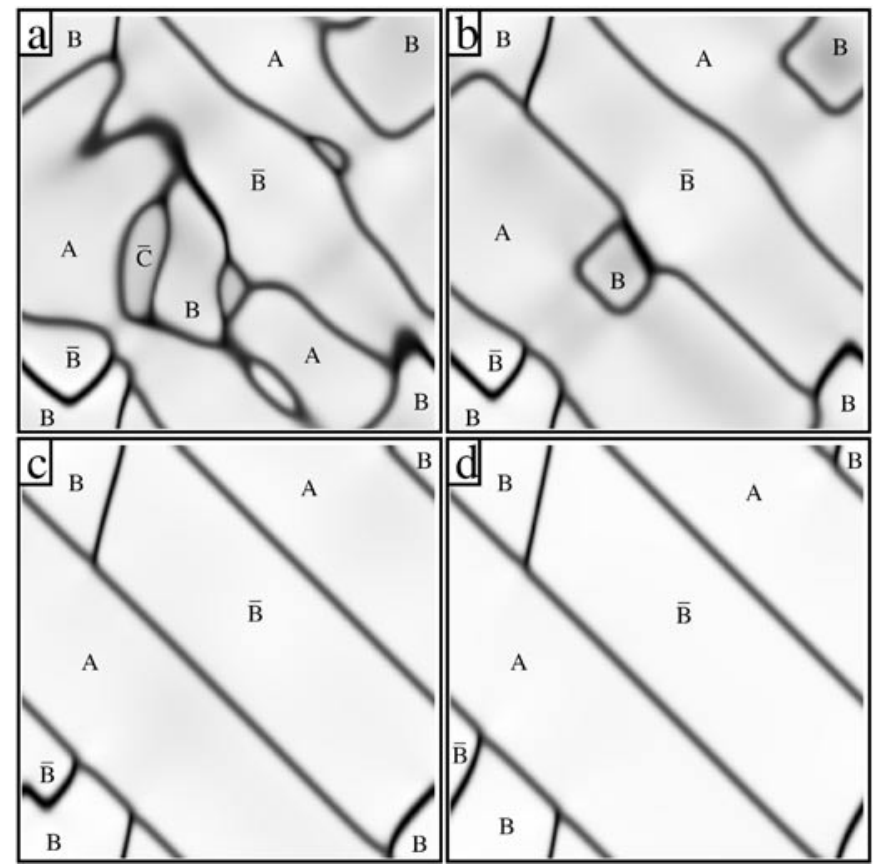

Figure 10. As figure 9, but at $c=0.44, \eta_{\max }^{2} \simeq 0.18$, and the following values of $t^{\prime}$ : (a) 110 ; (b) 140; (c) 200; and (d) 350 .

Frames 9(a)-(c) show that in the first stages of the evolution the proportion of conservative APBs with respect to non-conservative ones increases, due to the lower surface energy of the c-APBs. Later on, with the beginning of the tweed stage, the deformational effects become important leading to a dying out of both APDs $C$ and $\bar{C}$ and their c-flip APBs. However, the conservative shift-APBs within twin bands survive, and in the final frame 9(d) they are mostly 'step-like', consisting of (100)-type oriented conservative segments and small nonconservative ledges. These step-like APBs can be viewed as a 'faceted' version of tilted APBs discussed above and seen in figures 1-8. Such step-like APBs were observed under the $\mathrm{L} 1_{0}$ ordering of $\mathrm{CuAu}$ and some $\mathrm{CuAu}$-based alloys [7], and they are also similar to those observed under the $\mathrm{L}_{2}$ ordering in both simulations [15] and experiments for the $\mathrm{Cu}_{3} \mathrm{Au}$ alloy [30].

As was repeatedly noted in [16] and above, an increase of non-stoichiometry $\delta c=(0.5-c)$ or temperature $T$ leads to a sharp decrease of both the anisotropy of the APB energy and the energy preference of conservative APBs with respect to non-conservative ones. Therefore, with increase of $\delta c$ or $T$ the proportion of conservative APBs in transient microstructures falls off, and at sufficiently high $\delta c$ or $T$ such APBs are not formed at all under the transformation. This results in drastic microstructural changes of the evolution, including sharp, phase-transitionlike changes in the morphology of aligned shift-APBs within twin bands, from the 'faceting' to the 'tilting'. This is illustrated by figure 10 which shows the evolution of model 1 at a significant non-stoichiometry $\delta c=0.06$, and this evolution is qualitatively different from that for a stoichiometric alloy shown in figure 9.

Figure 11 illustrates the transition from the 'faceted' to the 'tilted' morphology of shiftAPBs within nearly equilibrium twin bands with variations of $T$ or $\delta c$ for model 2. An examination of the intermediate stages of transformations illustrated by this figure shows that the morphological changes are realized via some local bends of faceted APBs. This is 


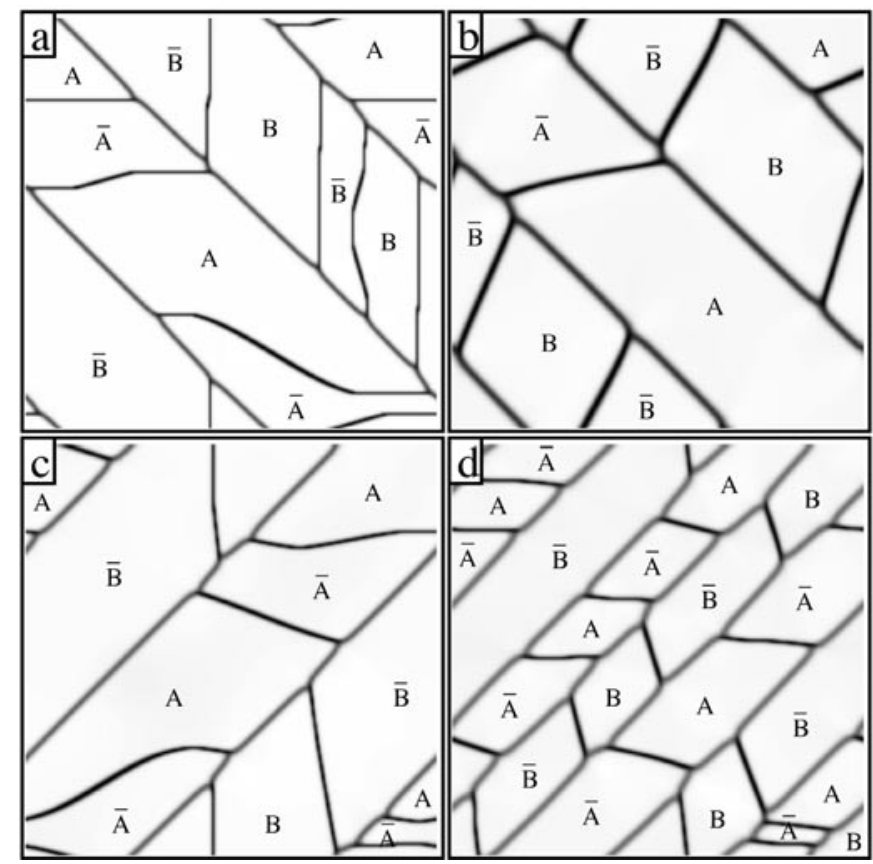

Figure 11. As figure 1, but for model 2 at $\left|\varepsilon_{m}\right|=0.1$ and the following values of $c, T^{\prime}, \eta_{\max }^{2}$, and $t^{\prime}:$ (a) $c=0.5, T^{\prime}=0.77, \eta_{\max }^{2} \simeq 0.24$, and $t^{\prime}=350$; (b) $c=0.5, T^{\prime}=0.95, \eta_{\max }^{2} \simeq 0.21$, and $t^{\prime}=300 ;$ (c) $c=0.46, T^{\prime}=0.77, \eta_{\max }^{2} \simeq 0.20$, and $t^{\prime}=350$; and (d) $c=0.44, T^{\prime}=0.77$, $\eta_{\max }^{2} \simeq 0.19$, and $t^{\prime}=300$.

also revealed by a comparison of frames 11(a), (c), and (d) with each other. Therefore, the 'morphological phase transition' mentioned above is actually smeared over some interval of temperature or concentration. However, frames 11(a)-(d) show that the 'intervals of smearing' of such transitions can be relatively narrow.

\section{Conclusions}

Let us summarize the main results of this work. To study microstructural evolution under $\mathrm{L}_{1}$-type orderings in alloys we first derive a microscopical model for the effective interatomic deformational interaction due to the so-called Kanzaki forces describing the interaction of lattice deformations with site occupations. It generalizes an analogous model of Khachaturyan for dilute alloys [10] to the physically interesting case of concentrated alloys. We take into account the non-pairwise contribution to Kanzaki forces, and the resulting effective interaction $H_{d}$ is non-pairwise, too, unlike in the case of dilute alloys. Assuming non-pairwise Kanzaki forces to be short ranged, we express the deformational interaction $H_{d}$ in terms of two microscopical parameters which can be estimated from experimental data on lattice distortions under phase transformations, and we present such estimates for the alloys Co-Pt.

Then we employ the earlier-described kinetic cluster field method $[15,16]$ to simulate $\mathrm{A} 1 \rightarrow \mathrm{L} 1_{0}$ transformation after the quench of an alloy from the disordered A 1 phase to the $\mathrm{L} 1_{0}$ phase for five alloy models with different types of chemical interaction. The transformation can be divided into three stages, in accordance with the increasing importance of the deformational interaction $H_{d}$ : the 'initial', 'tweed', and 'twin' stages. For the initial stage the deformational 
effects are insignificant, while for the tweed stage they become comparable with those of the chemical interaction $H_{c}$. For the twin stage, the tetragonal distortion of $\mathrm{L} 1_{0}$-ordered antiphase domains (APDs) leads to the formation of (110)-type oriented twin bands which include only two types of APD with the same tetragonal axis, and these axes in adjacent bands have alternate (100) and (010) orientations for the given set of (110)-type oriented bands.

The type of evolution strongly depends on the range $R_{\text {int }}$ of the chemical interaction. For systems with an extended or intermediate $R_{\text {int }}$ at both the initial and tweed stages we observe the following features: (a) abundant processes of fusion of in-phase domains; (b) a great number of peculiar long-living configurations, the quadruple junctions of APDs described in section 4; and (c) numerous processes of 'splitting' of an antiphase boundary separating the APDs with the same tetragonal axis ('shift-APB') into two APBs separating the APDs with perpendicular tetragonal axis (flip-APBs). The simulations also illustrate a sharp temperature dependence of the evolution, in particular, a notable increase of both the width of APBs and the characteristic size of initial APDs with elevating $T$. The deviation from stoichiometry affects the evolution similarly to temperature: for increase of both the non-stoichiometry $\delta c=(0.5-c)$ and $T$, all APBs thicken, while shift-APBs become less stable with respect to flip-APBs.

For the twin stage, our simulations reveal the following features of transient microstructures: (1) semi-loop-like shift-APBs adjacent to the twin band boundaries; (2) 'Sshaped' shift-APBs stretching across the twin band; (3) short and narrow twin bands ('microtwins') lying within the larger twin bands; and (4) processes of 'transverse coarsening' of twinned structures via a shrinkage and disappearance of some microtwins. All these features agree with experimental observations [2-4]. For the final, nearly equilibrium twin bands the simulations demonstrate a peculiar alignment of shift-APBs with a certain tilting angle between the band orientation and the APB plane, and this tilting angle sharply depends on the interaction type, particularly on the interaction range $R_{\text {int }}$. Such alignment of APBs seems to be observed in the CoPt alloy [1], and a comparison of experimental tilting angles with theoretical calculations [11] can provide information about the chemical interactions in an alloy.

A distinctive feature of the evolution for the short-range-interaction systems is the presence of many conservative APBs with the (100)-type orientation. The conservative shift-APBs are also present in the final twinned structures, and they are mostly 'step-like', consisting of (100)type oriented conservative segments and small non-conservative ledges. This can be viewed as a 'faceted' version of tilted APBs mentioned above. Such (100)-type alignment of shiftAPBs agrees with the observations for the alloy $\mathrm{CuAu}$ [7] for which chemical interactions are supposed to be short ranged $[15,30]$.

With increase of the non-stoichiometry $\delta c$ or temperature $T$, the energy preference of conservative APBs with respect to non-conservative ones decreases, and the proportion of conservative APBs in the microstructures falls off. This results in drastic microstructural changes, including sharp, phase-transition-like changes in morphology of aligned shift-APBs within twin bands, from their 'faceting' to the 'tilting'. Such 'morphological phase transitions' are actually smeared over some intervals of temperature or concentration, but the simulations show that the intervals of smearing can be narrow.

Finally, let us note that this and other studies of kinetics of multivariant orderings, such as the $\mathrm{D}_{3}, \mathrm{~L}_{2}$, and $\mathrm{L} 1_{0}$ orderings $[15,16,28]$, show that the microstructural evolution under such orderings reveals a great variety of peculiar features, the detailed form of which sharply depends on the type of interaction, temperature, the degree of non-stoichiometry, the scale of lattice distortion, and other factors. Therefore, detailed experimental and theoretical studies of this evolution can provide important information about details of microscopical interactions in alloys. 


\section{Acknowledgments}

The authors are much indebted to V Yu Dobretsov for help in this work; to N N Syutkin, V I Syutkina and Jean-Louis Bocquet, for valuable information about experimental data; and to Georges Martin, for numerous stimulating discussions. The work was supported by the Russian Fund of Basic Research under grants Nos 00-02-17692 and 00-15-96709.

\section{References}

[1] Leroux C, Loiseau A, Broddin D and Van Tendeloo G 1991 Phil. Mag. B 6457

[2] Zhang B, Lelovic M and Soffa W A 1991 Scr. Metall. 251577

[3] Zhang B and Soffa W A 1992 Phys. Status Solidi a 131707

[4] Yanar C, Wiezorek J M K and Soffa W A 2000 Phase Transformations and Evolution in Materials ed P E A Turchi and A Gonis (Warrendale, PA: The MMM Society) p 39

[5] Tanaka Y, Udoh K-I, Hisatsune K and Yasuda K 1994 Phil. Mag. A 69925

[6] Oshima R, Yamashita M, Matsumoto K and Hiraga K 1994 Solid-Solid Phase Transformations ed W C Johnson et al (Warrendale, PA: The MMM Society) p 407

[7] Syutkina V I, Abdulov R Z, Zemtsova N D and Yasyreva L P 1984 Fiz. Met. Metalloved. 58745

[8] Roitburd A L 1968 Fiz. Tverd. Tela 103619 (Engl. transl. 1969 Sov. Phys.-Solid State 10 2870)

[9] Khachaturyan A G and Shatalov G A 1969 Zh. Eksp. Teor. Fiz. 561037 (Engl. transl. 1969 Sov. Phys.-JETP 10 557)

[10] Khachaturyan A G 1983 Theory of Structural Phase Transformations in Solids (New York: Wiley)

[11] Vaks V G 2001 Pis. Zh. Eksp. Teor. Fiz. 73274 (Engl. transl. 2001 JETP Lett. 73 237)

[12] Chen L-Q, Wang Y Z and Khachaturyan A G 1992 Phil. Mag. Lett. 6515

[13] Vaks V G 1996 Pis. Zh. Eksp. Teor. Fiz. 63447 (Engl. transl. 1996 JETP Lett. 63 471)

[14] Belashchenko K D and Vaks V G 1998 J. Phys.: Condens. Matter 101965

[15] Belashchenko K D, Dobretsov V Yu, Pankratov I R, Samolyuk G D and Vaks V G 1999 J. Phys.: Condens. Matter 1110593

[16] Pankratov I R and Vaks V G 2001 J. Phys.: Condens. Matter 136031

[17] Faulkner J C, Wang Y and Stocks G M 1997 Phys. Rev. B 557492

[18] Rouchy J and Waintal A 1975 Solid State Commun. 171227

[19] Zunger A 1994 Statics and Dynamics of Alloy Phase Transformations (NATO ASI Series B: Physics, vol 319) ed A Gonis and P E A Turchi (New York: Plenum) p 361

[20] Beiden S V, Samolyuk G D, Vaks V G and Zein N E 1994 J. Phys.: Condens. Matter 68487

[21] Vaks V G, Beiden S V and Dobretsov V Yu 1995 Pis. Zh. Eksp. Teor. Fiz. 6165 (Engl. transl. 1995 JETP Lett. $6168)$

[22] Landolt-Börnstein 1990 Numerical Data and Functional Relationships in Science and Technology (Berlin: Springer) New Series Group III, vol 26 p 298

[23] Chassagne F, Bessiere M, Calvayrac Y, Cenedese P and Lefebvre S 1989 Acta Metall. 372329

[24] Vaks V G and Samolyuk G D 1999 Zh. Eksp. Teor. Fiz. 115158 (Engl. transl. 1999 Sov. Phys.-JETP 88 89)

[25] Berg H and Cohen J B 1972 Metall. Trans. 31797

[26] Leroux C, Cadeville M C, Pierron-Bohnes V, Inden G and Hinz F 1988 J. Phys. F: Met. Phys. 182033

[27] Ducastelle F 1970 J. Physique 311055

[28] Belashchenko K D, Samolyuk G D and Vaks V G 1999 J. Phys.: Condens. Matter 1110567

[29] Turchi P E A 1994 Intermetallic Compounds: Principles vol 1, ed J H Westbrook and R L Fleicher (New York: Wiley) p 21

[30] Potez L and Loiseau A 1994 J. Interface Sci. 291 